\title{
MASTERING MYCOLOGICAL MYSTERIES WITH EXPLORATIONS OF HARPELLALES ASSOCIATED WITH CULICIDAE AND OTHER DIPTERANS IN IDAHO
}

by

Michael McCormick

\author{
A thesis \\ submitted in partial fulfillment \\ of the requirements for the degree of \\ Master of Science in Biology \\ Boise State University
}

December 2018 
(C) 2018

Michael McCormick

ALL RIGHTS RESERVED 


\title{
BOISE STATE UNIVERSITY GRADUATE COLLEGE
}

\section{DEFENSE COMMITTEE AND FINAL READING APPROVALS}

\author{
of the thesis submitted by \\ Michael McCormick
}

Thesis Title: Mastering Mycological Mysteries with Explorations of Harpellales Associated with Culicidae and Other Dipterans in Idaho

Date of Final Oral Examination: $\quad 24$ April 2018

The following individuals read and discussed the thesis submitted by student Michael McCormick, and they evaluated his presentation and response to questions during the final oral examination. They found that he passed the final oral examination.
Merlin M. White, Ph.D.
Chair, Supervisory Committee
Ian C. Robertson, Ph.D.
Member, Supervisory Committee
Pete Koetsier, Ph.D.
Member, Supervisory Committee

The final reading approval of the thesis was granted by Merlin M. White, Ph.D., Chair of the Supervisory Committee. The thesis was approved by the Graduate College. 


\section{DEDICATION}

To my ever-enduring family for their endless support. 


\section{ACKNOWLEGDMENTS}

I would like to thank my undergraduate crew for thinking outside the box and taking gut fungi to new heights. Mathew (Teo) Geisler for his help in the field and with laboratory experiments. Christina Moschella for help with dissections and microscope pictures. Tony Berlin for his intense work ethic and seemingly endless data entry. Also, thank you to my fellow graduate students, Stephanie Coates and Britt Pendleton, for helping with figures and formatting. Skyler Swiecki, Christine Hayes, and Bryce Robinson for their help on presentations. They are true geniuses. The endless banter of my cohorts kept a smile on my face. I would like to thank Bill Bourland, MD, for his detailed instruction and use of his scanning electron microscope. Eddie Beard, at Clemson University for his insight with mosquitoes and work with Harpella melusinae midgut dwelling gut fungi. Laura Bond, who offered statistical help and wisdom. Thank you to Boise State University for the SRP award. My committee members for their knowledge and patience. Finally, Merlin M. White for giving me the freedom to explore and be independent. 


\section{TABLE OF CONTENTS}

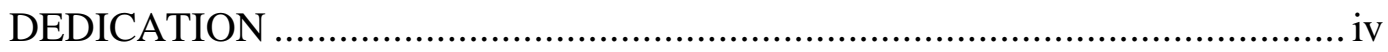

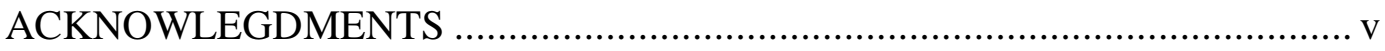

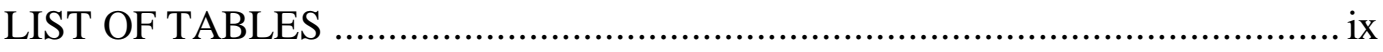

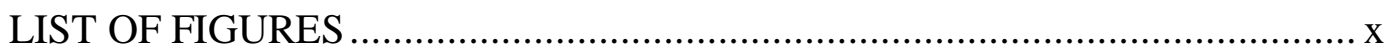

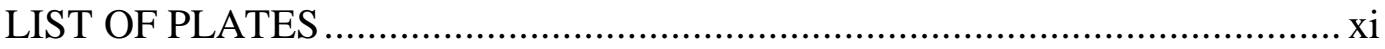

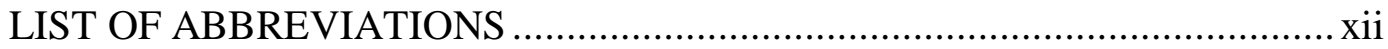

A SURVEY OF MOSQUITOES AND ASSOCIATED HARPELLALES GUT

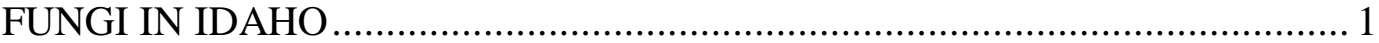

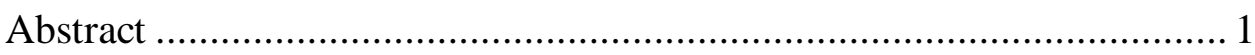

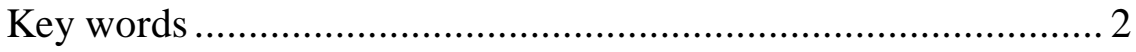

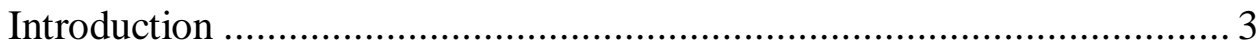

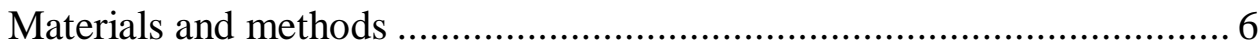

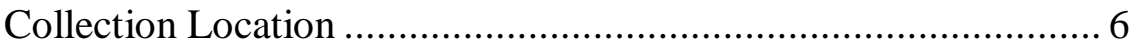

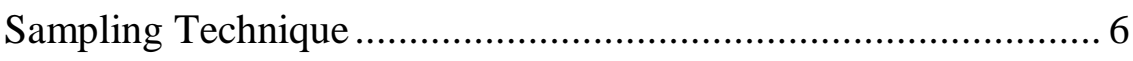

Adult and Larval Identification ................................................... 7

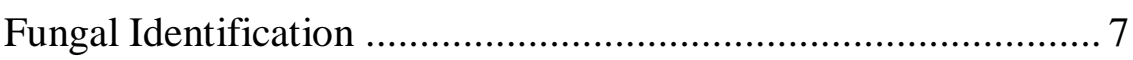

Mosquito Larvae Rearing and Inoculation Techniques.................. 9

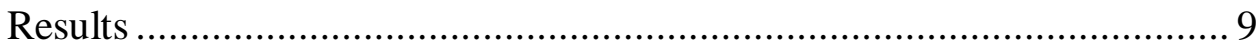

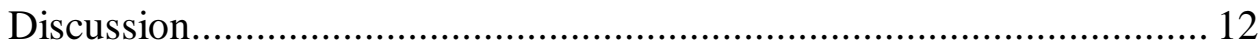

Spore Survival or Reservoir Host............................................. 13 
Vertical Transmission Via Cysts.............................................. 16

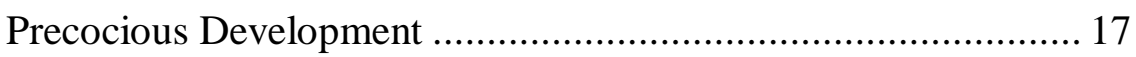

Physiological Misfiring or Adaptive Spore Release Stage ........... 18

Speculation on a Viral Connection............................................. 19

DISPERSAL ECOLOGY OF A GENERALIST GUT FUNGUS, ZANCUDOMYCES CULISETAE, AN OBLIGATE ENDOBIONT OF AQUATIC DIPTERA IN A MOUNTAIN STREAM ECOSYSTEM................................. 27

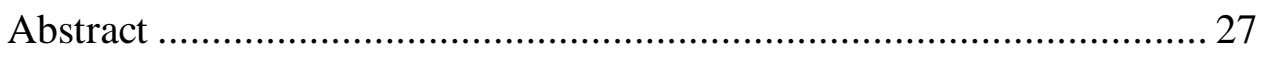

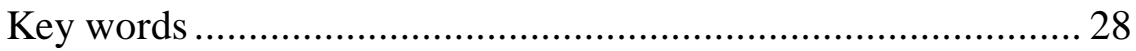

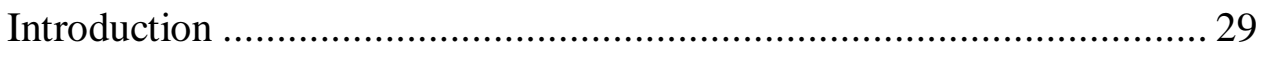

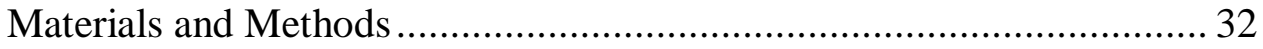

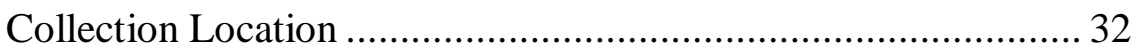

Collection Technique............................................................... 33

Artificial Containers ................................................................ 33

Laboratory Handling, Processing, and Vouchering ..................... 34

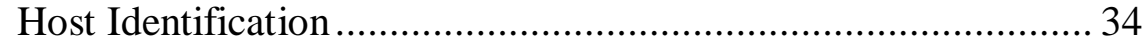

Fungal Identification .............................................................. 34

Spore Volume Comparison .......................................................... 36

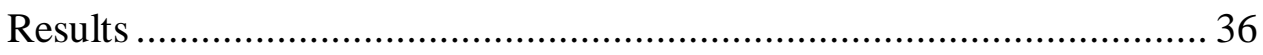

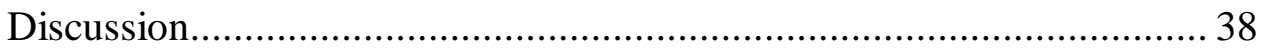

Adult Ovarian Cysts .................................................................. 40

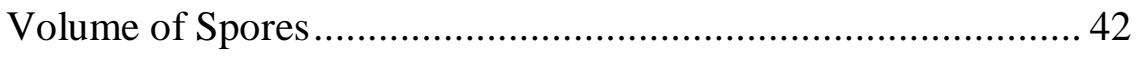

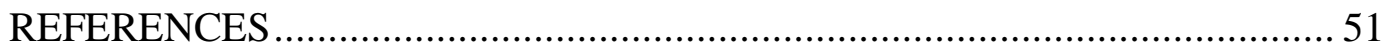

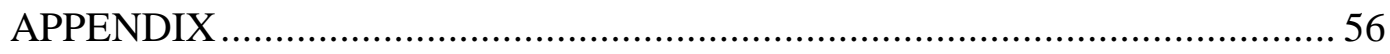


An extended table of survey results for Idaho

An extended table of Renwyck Creek collections and further observations ......................................................................... 62

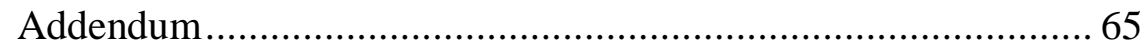




\section{LIST OF TABLES}

Table 1.1 Survey data for mosquitoes from all Idaho collection sites........58

Table 2.1 Survey data for dipteran collections from Renwyck Creek and confluences..........................................63 


\section{LIST OF FIGURES}

Figure 1.1 Rain data overlaid with topographic map and collection points...26

Figure 2.1 Map of Renwyck Creek collection sites......................44

Figure 2.2 Renwyck Creek flood zone with lentic mosquito habitat.........45

Figure 2.3 Renwyck Creek splash zone depicting solitary midge habitat......46

Figure 2.4 Renwyck Creek spring boil zone, Chiromomidae habitat.........47

Figure 2.5 Density plot of Zancudomyces culisetae trichospores.............48 


\section{LIST OF PLATES}

Plate $1.1 \quad$ Image of Culex sp. mosquito larva.........................21

Plate 1.2 Images of Smittium culicis, Smittium minutisporum,

Zancudomyces culisetae, and precocious germlings ............22

Plate 1.3 SEM image of Zancudomyces culisetae microstructure............23

Plate 1.4 SEM image of Zancudomyces culisetae 24

Plate 1.5 SEM image of Zancudomyces culisetae collar and appendage....25

Plate 2.1 Image of Zancudomyces culisetae with holdfast.................49

Plate 2.2 Image of Zancudomyces culisetae, Nomarski Interference

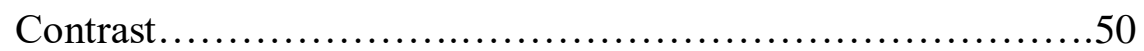




\section{LIST OF ABBREVIATIONS}

$\begin{array}{ll}\text { BHI } & \text { Brain Heart Infusion } \\ \text { C } & \text { Celsius } \\ \text { ID } & \text { Idaho } \\ \text { MM } & \text { Michael McCormick } \\ \text { N } & \text { No } \\ \text { NIC } & \text { Nomarski interference contrast } \\ \text { SW } & \text { South West } \\ \text { TG } & \text { Mathew (Teo) Geisler } \\ \text { W } & \text { Merlin M. White } \\ \text { Y } & \text { Yes }\end{array}$




\title{
A SURVEY OF MOSQUITOES AND ASSOCIATED HARPELLALES GUT FUNGI \\ IN IDAHO
}

\begin{abstract}
Presented is the first field survey and laboratory-based study focused on Harpellales gut fungi found in Culicidae (mosquito) larvae collected from various parts of Idaho. Overall, 34 sites were sampled ranging from urban storm drains and irrigation puddles to pristine stream-side puddles. These sites yielded 17 different species of mosquitoes and three previously described species of gut fungi. Three species of mosquitoes were the first recorded observations as hosts of the following gut fungi: Culiseta alaskaensis was infested with Zancudomyces culisetae (from Renwyck Creek), Culex tarsalis with Smittium culicis (from Cottonwood Creek), and Ochleratus sp. with Smittium minutisporum (from Bear Creek). Although unknown as to why, only one type of mosquito-borne fungus was found in each creek's nearby lentic system. This finding warrants future investigation. Arid conditions throughout the state led to challenges in finding infested hosts. However, gut fungi were observed in larvae when their lentic sites had been directly connected with a stream system. Lentic systems free of any lotic association did not yield any gut fungal infestation. To test the susceptibility of isolated lentic sites, non-infested storm drain mosquitoes, Culex pipiens, were collected, inoculated, and colonized by Z. culisetae in the laboratory. The survey also yielded the first natural observation of both $S$. culicis and Z. culisetae as precocious midgut dwelling germlings found in Culicidae larvae. The germlings of both species were either simply
\end{abstract}


attached to the surface or actively penetrating the midgut. When penetrating, each germling had distinct forms of attaching and anchoring during precocious development, which were different from a more typical "gluing" and non-penetrating holdfast attachment in the hindgut. These penetrations created openings in the midgut, which left mosquito larvae vulnerable to viral passage into the hemocoel. The possibility of germlings participating in vertical transmission of viruses into adult stages is discussed speculatively.

$\underline{\text { Key words }}$

Harpellales, life history, symbiosis 


\section{Introduction}

In many regards mycology and symbiosis have numerous niches left to be explored. Of animal associated fungi, found in the currently informal classification zygomycetes II, the Kickxellomycotina are a group of two orders: Asellariales and Harpellales (Lichtwardt, 1986; Spatafora et al., 2016). These orders once belonged to a group now informally referred to as the trichomycetes or "hair like" fungi (Hibbett et al., 2007). In their most commonly known form, species of Harpellales consist of a gut attaching holdfast, and develop into branched or unbranched spore producing thalli with generative cells that produce asexual trichospores. Some, after conjugation (nuclear exchange) of mature fertile thalli, produce sexual zygospores from zygosporophores. Species of Harpellales mature in the digestive tract of the immature aquatic stages (larvae or nymph) of arthropods, except for one species of freshwater isopod (Lichtwardt et al., 1986, 2004). There, they grow and produce sexual or asexual spores that are released through defecation into the environment for ingestion by another suitable host. These endobionts follow the full spectrum of symbiosis, ranging from parasitism to mutualism; however, they are most often considered commensalistic (Misra and Horn, 2001). Interactions of gut fungi and their varied hosts have been well documented worldwide (Lichtwardt et al., 1986, 2004, 2007). The ecology and life history between obligate endosymbiotic gut fungi (Harpellales) and mosquitoes (Diptera: Culicidae) forms a distinctive association. However, this relationship between gut fungi and mosquitoes has not been the focus of any previous extended studies in Idaho. The present field-based survey establishes a widespread baseline account of Harpellales gut fungi found in mosquitoes in varying locales throughout the state. 
Worldwide, mosquitoes are well recognized as hosts for multiple species of gut fungi, including: Smittium culicis, S. insulare, S. minutisporum, S. morbosum, S. simulii, and Zancudomyces culisetae (Lichtwardt, 2007). Until recently revised taxonomically, Z. culisetae had been recognized as a species of Smittium (Wang et al., 2013). Prior survey-based studies of gut fungi have yielded new taxa, expanded distribution ranges of known species, and new hosts of harpellid fungi (Bench and White, 2012; Lichtwardt, 1986; Valle and Santamaria, 2004; White et al., 2006). The success of those studies and the limited collections of Culicidae larvae in Idaho, left this region wide open to be explored.

I hypothesized that field-collected larval mosquitoes from any type of lentic system, regardless of the origins of water source (e.g. irrigation, rain, well, or creek), should contain Harpellales gut fungi in their digestive tracts. This hypothesis is based on the prediction that an adult cyst stage exists and vertically transmits these microorganisms from one site to another, including disconnected lentic habitats. As an example, adult black fly (Simulium noelleri) ovaries may become infected with fungal Harpella melusinae cysts (chlamydospores), which once laid on or near egg masses, produce trichospores or zygospores that are consequently consumed by newly hatched black fly larvae (Moss and Descals 1986; Moss and Taylor 1994; Moss 1998; Labeyrie et al. 1996). Developmentally synchronized, these asexual or sexual spores are available to infest the next generation of larval black flies (Rizzo and Pang, 2005).

The mosquito season in SW Idaho typically spans from April to November. Peak larval activity is in late July, with numbers declining gradually until the first hard freeze in October/November (personal observations). The climate is classified as arid or semi- 
arid steppe (Kottek et al. 2006). Aridness becomes the limiting factor for lentic sources of larvae because spring puddles are lost in early May due to evaporation, unless supplied through outside sources such as irrigation, wells, or lotic systems. Each successive search for mosquitoes built on previous assessments as well as lab-based results and prompted subsequent refinements in collecting. I had to understand the local climate, how flowing sources of water provided lentic habitats, and where to find sustained lentic systems, for this project to continue to move forward.

During the present study, discovered lentic systems considered as "suitable habitats" for mosquito larvae were those found to be maintained by a continuous source of water. These habitats included storm drains and puddles along landscaped grounds filled with irrigation, rain, or well water. In more natural areas, suitable habitats included puddles, which were maintained by nearby creeks. During spring, areas of creeks and rivers are often carved out and left as standing puddles after the high water of melting snow recedes. These puddles are kept hydrated through underground infiltration of porous sand and silt by nearby stream water.

Despite many earlier studies and records, the complete life history and symbiosis of gut fungi-mosquito association is still not fully understood, especially as they might relate to mosquitoes as vectors of disease. This study was also undertaken with an interest to note any evidence of a possible link between gut fungi and pathogen transfer, as mosquitoes are significant to humans as vectors of disease. Overall, this survey expands the knowledge of mosquito ecology as it relates to Harpellales gut fungi infestation in SW Idaho. It is hoped that these observations and data may be more broadly applied and considered in future research endeavors, both in the state and beyond. 


\section{Materials and methods}

\section{Collection Location}

Mosquitoes were collected in multiple counties, mostly in SW Idaho. Ada county was the one most sampled during the 2015 and 2016 seasons (Table 1.1 and Figure 1.1). I started collecting when mosquito larvae began hatching in April and continued collections through November during both years, with additional but sparse collecting in 2017. Latitude and longitude of each collection site was determined using Google Maps and collection logs (Table 1.1).

\section{Sampling Technique}

Mosquito larvae were collected using a small handheld fish net. Samples taken at the same site were pooled and stored in a 1 gallon Ziplock ${ }^{\circledR}$ bag. Nets and storage bags were not reused, to prevent cross contamination between collection sites. Water, leaves, debris, and detritus also were gathered at each site and consolidated within the bag that contained the larvae. Bagged samples were placed in a cooler with ice and transported back to the laboratory. Larvae were stored at $4^{\circ} \mathrm{C}$ for up to two weeks until dissection. When transferring larvae from the sampling bag to microscope slide for dissection, a unique sterile pipette was used with each sample to prevent cross contamination.

Some of the more permanent lentic systems were sampled multiple times throughout the season. A unique sampling bag was used for each collection date. Each distinct lentic system collection was assigned a site number. Individual collections were labeled with date, temperature, location, and a unique collection code. 


\section{Adult and Larval Identification}

Several hosts from each collection were preserved in glass vials with $2 \mathrm{ml}$ of $70 \%$ ethanol as voucher specimens, to be held at Boise State University in Dr. Merlin White's trichomycete laboratory. Vouchers were labeled according to collection, site, date, and species. Larvae and adults were identified by their morphology using the regional identification key in Brothers (2003).

\section{Fungal Identification}

Mosquito larvae were dissected in their $4^{\text {th }}$ instar. Often, $3^{\text {rd }}$ instars were collected and allowed to reach $4^{\text {th }}$ instar while in the sample bag at $20^{\circ} \mathrm{C}$ with a $15 \mathrm{hr}$ light cycle. At least five larvae were dissected from each collection that was positive for infestation. Twenty larvae were dissected from each collection where no infestation was present. On subsequent visits to a site, an additional 20 larvae were dissected. After placing the larva on a microscope slide in a drop of sterile $\mathrm{diH}_{2} \mathrm{O}$, the head of the larva was removed with sharpened jeweler forceps. The saddle/anal segment was then removed, leaving the siphon attached to the host, but extracting the hindgut and midgut with the saddle (Plate 1.1). Next, the hindgut and midgut were separated at the pyloric sphincter, after which the hindgut was transferred to a fresh drop of sterile $\mathrm{diH}_{2} \mathrm{O}$ on a microscope slide. The midgut was given approximately one minute to allow for the food bolus and epithelial layer to separate via osmotic pressure from the $\mathrm{diH}_{2} \mathrm{O}$, then the transparent peritrophic matrix was placed into the same drop as the hindgut. The hindgut was lacerated with forceps that had been sterilized with ethanol and flame to prevent contamination from previous dissection. A glass cover slip was gently placed over the wet mounted specimen, using capillary action of the $\mathrm{diH}_{2} \mathrm{O}$ to eliminate air bubbles. 
Using a Nikon Eclipse 80i microscope, each slide was examined for Harpellales gut fungi by using a linear back and forth motion at 200x magnification until the entire area of the cover slip was examined. If fungi were present, whether just loose spores, or with dense thalli, the slide was labeled with the date, collection number, initials, serially increasing code, and preserved in lactophenol cotton blue (LCB). The glass cover slip was double sealed with Revlon brand nail enamel (clear 771) (Lichtwardt et al., 1986, 2004; Bench and White, 2012). Released, free floating spores collected from the hindgut were measured for length, width, and collar size. Averages were then compared to morphometric data of known samples and were used to delineate between species of gut fungi. Overall measurements, plus qualitative assessment, including spore length, where it was widest (i.e. medial), and collar (generative cell attachment) shape, when considered together as a group, provide ample data for identification purposes (Lichtwardt, 2007). Spot Advanced software (4.6) was used for spore measurements and collar morphology of captured images, which were taken with a 2 MP Spot Color Mosaic camera (Diagnostic Instruments, Sterling Heights, Michigan).

Axenic culture attempts were made for each species of Harpellales, undertaken with brain heart infusion (BHI) agar, sterile $\mathrm{diH}_{2} \mathrm{O}$ overlay in $60 \mathrm{~mm} \times 15 \mathrm{~mm}$ petri dishes, and added $<1 \mathrm{~mL}$ of 40,000 units of Penicillin G and 80,000 units of Streptomycin sulfate antibiotics per milliliter (Lichwardt et al, 1986, 2004, chapter 3).

Scanning electron microscopy was used to reveal finer details of the microstructure of an isolate Z. culisetae (ID-333). Samples were fixed in a solution of $2.5 \%$ glutaraldehyde and $1 \%$ osmium tetroxide (final concentrations), dehydrated in a graded ethanol series, dried in an EMS850 critical point dryer and gold-coated in a Cressington 
108 sputter coater ( 0.15 mbar Argon, 7mA, 40s, and 15 cycles). Specimens were examined in a Hitachi SU-3500 scanning electron microscope $(10.0 \mathrm{kV}, 5.6 \mathrm{~mm} \mathrm{M}-\mathrm{x} 16.0 \mathrm{k}$ $\mathrm{SE})$.

Mosquito Larvae Rearing and Inoculation Techniques

A new axenic culture of Z. culisetae (ID-333) was grown in BHI media with sterile water overlay without antibiotics and allowed to grow for two weeks at room temperature. Four $\mathrm{mL}$, at 70,000 spores per $\mathrm{mL}$, of $Z$. culisetae culture were transferred to Culex pipiens (ID-377) kept in $300 \mathrm{~mL}$ of their indigenous storm drain water (see Wilson [2013] appendix C for details on spore calculation methods). Larvae were reared at room temperature $\left(20^{\circ} \mathrm{C}\right)$ with a 15 -hour daylight cycle, fed a mixture of 50/50 brewer's yeast/liver powder (NOW®), and then dissected at $4^{\text {th }}$ instar. As a control, half of ID-377 larvae were not inoculated with Z. culisetae, for infestation comparison purposes (Table 1.1). Storm drain water consisted mostly of well-water run-off from Julia Davis Park, Boise, ID near where vegetation had been watered (per communication with Boise Parks and Recreation Department).

\section{Results}

From a total of 53 collections (Table 1.1), three previously described harpellid fungi were found during the 2015-17 survey of mosquito larvae in Idaho: Smittium culicis, S. minutisporum, and Z. culisetae. Zancudomyces culisetae, ID-333-MM1 was successfully isolated in axenic culture. This study is the first case where Culiseta alaskeinsis was infested with Z. culisetae, Culex tarsalis was infested with S. culicis, and Ochleratus sp. with S. microsporum. Culex sp. (excluding C. pipiens) (site 9, 17), C. tarsalis (site 2, 18), Culiseta sp. (site 18,21), and Ochleratus sp. (site 32, 34) presented 
without gut fungi infestation some of the time. Species of Culex (excluding C. pipiens) harbored both S. culicis and Z. culisetae, although at separate locations (site 9, 14). Anopheles spp. (site 2, 3), Coquillettidia sp. (site 30), and Culex pipiens (site 1, 5, 7, 8, $10,11,12,13,20,31$, and 33) never presented naturally with gut fungi infestation. A complete list of the mosquitoes sampled at Idaho sites between 2015-2017, and any associated gut fungi is presented in Table 1.1 of the appendix. Locations and number of sites sampled were also overlaid with rainfall data (Fig. 1.1). Mosquito species collected varied among the collection sites. Culex pipiens was most often found in storm drains and puddles near Boise, ID, whereas Culiseta spp. and Ochleratus spp. were collected in more pristine stand-alone puddles in late spring, or high elevation puddles near streambeds in late summer.

Puddles associated with streams in the form of pooled run off, or left behind after receding high water, yielded gut fungi upon dissection of collected larvae. By contrast, puddles and storm drains that were isolated from stream sources did not yield any gut fungi, even with subsequent repeated collections made throughout the mosquito season. However, when Culex pipiens (ID-377) larvae collected from those storm drain systems were inoculated in vitro with Z. culisetae (ID-333), the gut fungus grew readily in the hindguts of all dissected $4^{\text {th }}$ instar larvae. Control larvae from the same site and experiment were not infested.

Smittium culicis had trichospores that were medially swollen with a campanulate collar, had an average spore length $( \pm \mathrm{SE})$ of $21.4 \pm 0.2 \mu \mathrm{m}(\mathrm{n}=51$, range $=16.4-24.0$ $\mu \mathrm{m})$; average spore width $( \pm \mathrm{SE})$ of $6 \pm 0.08 \mu \mathrm{m}(\mathrm{n}=51$, range $=4.8-7.3 \mu \mathrm{m}) ;$ and average collar size $( \pm \mathrm{SE})$ of $7 \pm 0.1 \mu \mathrm{m}(\mathrm{n}=51$, range $=5.2-9.6 \mu \mathrm{m})$. 
Smittium minutisporum had too few spores for morphometric data analysis and should be considered a tentative identification. Its medially swollen spores, short length, and short cylindrical collars are common with its morphology.

Asexual spores of $Z$. culisetae were sub-medially swollen with a cylindrical collar, an average spore length $( \pm \mathrm{SE})$ of $16 \pm 0.1 \mu \mathrm{m}(\mathrm{n}=97$, range $=13.5-18.8 \mu \mathrm{m})$; average spore width $( \pm \mathrm{SE})$ of $3.8 \pm 0.05 \mu \mathrm{m}(\mathrm{n}=97$, range $=2.8-4.9 \mu \mathrm{m})$; and average collar size $( \pm \mathrm{SE})$ of $1.5 \pm 0.04 \mu \mathrm{m}(\mathrm{n}=97$, range $=0.8-2.6 \mu \mathrm{m})$.

Dissection of all mosquito larvae collections $(\mathrm{N}=16)$ infested with S. culicis and Z. culisetae in the hindgut, also revealed precocious midgut-dwelling fungi piercing the peritrophic protein matrix of its host (Table 2.1). Attempts to grow the precocious fungi were successfully undertaken by placing the gut lining and attached, unbranched, and non-sporulating germlings in a $60 \mathrm{~mm}$ culture plate with antibiotics and daily monitoring. These germlings further developed into mature fertile branched thalli and the produced trichospores morphometrically matched to Z. culisetae. Precocious gut fungi found in mosquitoes infested with S. culicis were not successfully cultured. The precocious thalli, in both genera, resembled an extruded sporangiospore and either with a long, thin extension (Z. culisetae) or hook (S. culicis) penetrated the peritrophic matrix (Plate 1.3). The extension and hook, internal to the protein matrix/lining, are quite unlike how and where these genera more typically attach, in the hindgut, where it produces a simple, nonpenetrating holdfast, with minimal glue-like substance to hold itself in place (Plate 1.2).

The temperature of puddles that had gut fungi infestation ranged from $7^{\circ} \mathrm{C}$ to $31.1^{\circ} \mathrm{C}$. These are the lowest and highest temperatures of any lentic site collected from in this study. The lowest temperature puddle was from a lentic site associated with Renwyck 
Creek and mosquito Culisetae alaskaensis (ID-333). The highest temperature was collected from a small tributary flood zone near the North Fork of the Boise River (ID315). Both genera of larvae taken from these puddles were infested with $Z$. culisetae (Table 1.1).

\section{Discussion}

Gut fungal infestations of $4^{\text {th }}$ instar mosquito larvae were associated only with puddles either connected to creeks in the form of run-off or those that formed below previous high-water events. This result was counter to my prediction that infestation would occur in any lentic systems where Culicidae larvae dwelt and in contrast to the prevailing notion from previous studies. Throughout the world, Harpellales found in mosquito larvae have been collected from many unusual places, including discarded trash along a roadway, vases, dog dishes, rock pools, and other seemingly isolated or disconnected habitats (Lichtwardt, 2004). In contrast, Idaho lentic sites have exhibited dependence on stream systems for trichospore inoculation.

Although it is rarely reported when no gut fungal infestation is found, there is one good example. Tuzet et al. (1961) dissected Aedes geniculatus and A. berlandi (Diptera: Culicidae) and found these tree-hole mosquitoes to be void of any gut fungi. However, in vitro inoculation of Smittium culicis in the tree-hole mosquito's source water resulted in successful infestation. Admittedly, these mosquitoes and tree-hole habitats are unlike anything from this study except that they too are isolated from stream systems. In Idaho, similar outcomes were seen with Culex pipiens (Diptera: Culicidae) found in storm drains and which did not yield any gut fungal infestation during either the 2015 or 2016 field seasons. The surprise and consistency of uninfested larvae prompted the question of 
whether isolated sites could become infested. To test the ability of storm-drain collected C. pipiens to become infested, I used specimens from the most larvae-populated storm drain (ID-377) that had been encountered. I predicted that either the host or the stormdrain water was preventing, or unsuitable for, gut fungi growth. However, after in vitro inoculation with $Z$. culisetae, $C$. pipiens in the same storm-drain water were readily infested in the laboratory. This weakens the idea that all gut fungi can be consistently distributed, and hosts inoculated, by an ovarian cyst stage. Minimally, there may be cues that trigger cyst development not yet understood (e.g., seasonality).

Spore Survival or Reservoir Host

The semi-arid nature of SW Idaho may yield the most likely explanation for the non-uniformity of Harpellales in Culicidae larvae. In this region, spring puddles that are not connected to streams or irrigation remain dry for extended periods during the hot summer months. This local dry period ( $\sim 5 \mathrm{mo})$ may inhibit the ability of gut fungi asexual spores to survive into the next season. Williams (1983) demonstrated that dry soil kept at $4^{\circ} \mathrm{C}, 10^{\circ} \mathrm{C}$, and $21^{\circ} \mathrm{C}$ for 5 months, once rehydrated, did not promote fungal infestation in mosquito larvae. However, soil that was kept moist under the same temperature conditions and duration provided the necessary elements for spore survival (Williams 1983). Williams' (1983) spore longevity experiments were designed to mimic Nebraska winter conditions where mosquito habitat either dries up or freezes during winter months, no longer supporting larval populations. His experiments provided an explanation for heavy infestation of larvae early in the spring of 1983. In a drier and contrasted example, vernal or spring ephemeral pools having infested mosquitoes were reported after a winter drought (Misra and Horn, 2001). Misra and Horn (2001) postulated that there must be 
some source other than over-wintering of trichospores to explain early infestation of larvae, especially considering that dry-cold conditions were not successful in Williams' in vitro experiments (1983). Thus, began the search for an adult insect vector and cyst stage of gut fungi.

The pattern described above concurs with the present study. With one exception, no SW Idaho lentic system that also underwent a period of drought, hot or cold, had mosquitoes that were infested. Cottonwood Creek (site 2) forms vernal and ephemeral palustrine-like overflows that dry up in early May and remain dry through the rest of the season. However, when Cottonwood Creek floods in early spring, it forms large shallow puddles under the riparian habitat that consistently yielded Culex tarsalis larvae heavily infested with Smittium culicis. These observations suggest that creeks might harbor gut fungi infested hosts that serve to increase spore loads and fungal cycling, which may increase the likelihood of inoculation of seasonal puddles left behind after such overflow.

To further explore the idea that creeks act as lotic reservoirs for gut fungi that can extend to inoculate additional lentic hosts, other dipterans such as midges should be studied in stream systems that form flood zone, mosquito bearing habitat. In one example, midges infested with $S$. culicis were described in lotic systems of Qinling mountains in western China (Strongman et al., 2010). However, lotic and lentic interactions may present challenges in finding infested hosts locally. Cottonwood Creek lotic systems had been sampled previously for over a year (2007-08), during which Chironomidae larvae did not reveal any $S$. culicis infestation, although the study did note a "S. culicis"-like species based on its morphology (Bench and White, 2012). Smittium 
culicis is one of the most morphologically variable of the harpellids and may, pending genetic analysis, be a species complex.

Even in lentic systems that did not experience drought, successful infestation of mosquito larvae by gut fungi also was dependent on being connected to a nearby lotic system, such as a creek, spring, or brook. When such a connection did not exist, such as with mosquitoes collected from storm drains, no dissected mosquito larvae had Harpellales. However, when indigenous water and uninfested Culex pipiens (ID-377) larvae from these storm drain systems were brought into the lab, larvae were readily infested with Z. culisetae from native Idaho axenic cultures (ID-333). Thus, neither Culex pipiens nor the storm drain water were inhibitory for fungal infestation. There was also a small ephemeral pond, disconnected from Cottonwood Creek, but just 100 meters beyond it, which yielded no gut fungi in Culex tarsalis (site 35). These examples suggest that the ability of fungi to survive and infest mosquitoes in SW Idaho requires association with lotic systems. These results differ from Lichtwardt's (1963) unpublished data book findings of infested mosquito larvae collected from discarded trash along a roadside near Pali lookout, Hawaii, USA (HAW 1-21). The main difference between these locations is the monsoonal climate of Hawaii (Kottek et al., 2006). Thus, when surveying an area, regional climatic conditions should be considered while judging potential spore survivability and predicting gut fungi distributions amongst candidate collection sites.

Williams (1983) noted that adjustments to experimental protocols are needed to better match specific environmental habitats necessary for spore survival. As Misra and Horn (2001) point out, Williams' (1983) experiments do not fully explain how spores survive the dry winter drought conditions found in many habitats where gut fungi 
infestations occur early in the season. It may be that there are other factors in streams and ephemeral puddles that support gut fungi spore longevity. For example, Williams (1983) discussed a report by Evans (1965) who found that root bark allowed for basidiospores to survive 18 months buried in soil, whereas no experimental trials survived without bark. It may be possible that forms of detritus, algae, exuviae, or leaf matter found in puddles may harbor critical elements for trichospore survival outside the host during winter months, which are commonly found in mosquito habitat. Hong et al. (1997) developed a model for explaining entomopathogenic and phytopathogenic spore longevity in relation to its specific environment. However, in the model there was variation in response both within and between species. He noted that further research is needed to better understand and predict a specific strain's spore survival response to temperature and humidity (Hong et al., 1997). In vitro experimentation with gut fungi spores and various detritus, puddle debris, and shed host exuvia should be studied further to help elucidate the life history of the Harpellales.

\section{Vertical Transmission Via Cysts}

Putative adult ovarian cyst stages in Culicidae should be further investigated, especially in connection with Chironomidae, as they are often found in the same lentic habitats wriggling around and feeding together (personal observation). This cohabitation may be important for understanding the life history and migration of gut fungi in Culicidae larvae from one lentic site to the next. Could these midges be the source of adult cyst vertical transmission? Williams attempted more than once to find an adult cyst stage, dissecting $~ 350$ adult mosquitoes after larval infestation, and yet no structural resemblance of any gut fungi was confirmed in later pupal or adult stages (Williams, 
1983; Misra and Horn, 2001). Though not definitive, his lack of evidence for cysts in mosquitoes lends credence to the notion that other hosts could be reservoirs for asexual or sexual spores in immature stages or, indeed, also develop and carry ovarian cysts to new sites. Moss (1998) has noted chlamydospores [ovarian cysts] of Smittium sp. on oviposited eggs of a Chironomus sp. (Chironomidae) (1998). Thus, if the symbiotic system, for any fungal species, could utilize more than one host, for example, midges being the source of Harpellales in some lentic systems cohabited by mosquitoes, this would be a huge advantage from a fungal perspective.

\section{$\underline{\text { Precocious Development }}$}

Precocious development is presumed to be a premature fungal growth occurring in the midgut of the host's digestive tract. Specifically, the sporangiospore (early germling) releases from a trichospore (monosporous sporangium or outer protective casing) that establishes precocious attachment and non-fertile thalli growth in this more anterior region of the gut. Normally, the sporangiospore would release and grow mature fertile thalli in the hindgut (Lichtwardt et al., 1997). Lichtwardt et al. (1997) reported such premature development of Smittium spp. in Chironomidae larvae, with fungal thalli piercing the peritrophic matrix of the midgut. They fed Orthocladius sp. larvae cultures of Smittium longisporum in vitro and upon dissection witnessed trichospores extruding, attaching, and or penetrating the protein matrix of the midgut (Lichtwardt et al. 1997). In my study, observations did not reveal the timing of Z. culisetae extrusion, attachment, and or penetration of the midgut lining. However, I removed and grew precocious extrusions, still attached to mosquito midguts, and these unbranched thalli elongated and matured into branched sporulating forms of $Z$. culisetae. In this case, the penetrating 
nature of the precocious thalli was unusual compared to its attachment and normal development where it uses glue and a simple holdfast to attach in the hindgut (Lichtwardt, 2004).

\section{Physiological Misfiring or Adaptive Spore Release Stage}

Can a change in $\mathrm{pH}$, whether in the midgut or stream water, cause some spores to prematurely extrude and anchor in the peritrophic matrix? In vitro experiments by Horn (1989) have shown that there are two phases for spore extrusion. Highest percent extrusion success was obtained when trichospores were first exposed to Phase 1 at $\mathrm{pH} 10$ with potassium ions concentration $\geq 20 \mathrm{mM}$, which mimicked the midgut, and then to the hindgut treatment of Phase II, which lowered the $\mathrm{pH}$ to 7 (Horn 1989). It is possible that premature extrusion may result if the host's midgut was not able to compensate for environmental changes in $\mathrm{pH}$.

During dissections of mosquito larvae, I found many components of shed exuviae (insect exoskeletons) in the digestive tract. These components may have included precocious extrusions (personal observations). It is possible that penetrated immature thalli attach to the next host, mature and successfully release spores, thereby perpetuating its life cycle. As compared to spores being eaten by a late instar insect, an alternative strategy of precocious development may play a role in spore success if the host molts before fungal maturation. The second host may become colonized faster by gut fungi if spores are already exuded. If the extrusions arrive in late instar, the already exuded spores could provide an advantage to the fungus in producing more asexual trichospores. Future research should consider whether spores might not be more strategically placed along the midgut of the digestive tract, as opposed to simply being "misfired" reproductive 
propagules. To test this possibility, researchers could feed penetrating precocious extrusions stuck in a midgut to sterile mosquitoes and determine whether infestation results. This experiment should be compared to an additional experiment where spores are simply added to the water for larval consumption.

$\underline{\text { Speculation on a Viral Connection }}$

Harpellid precocious penetrations of the sort noted here have not been considered as a possible explanation for viral transfer into mosquito adulthood. Anderson and Main (2006) collected wild mosquito larvae and demonstrated vertical transmission of West Nile virus in the laboratory. However, they offered no evidence of infestations of Harpellales in collected larvae. Having established that vertical virus transmission does occur, the next step would be to consider how it occurs. Penetrating gut fungi have the potential to allow access beyond their host's protective protein matrix. This protein matrix consists of increased levels of actin protein in areas of muscle attachment where the water temperatures are below $18^{\circ} \mathrm{C}$. How this affects the gut fungus is unknown, but it may be pertinent to rates of fungal penetration of the midgut (Mijung et al. 2006). Consumption of virally contaminated detritus or water may allow for (vertical) transmission of viruses into the hemolymph via the hole created by the precocious gut fungi. Under such conditions, the virus might subsequently have the opportunity to stay within the infested larva through pupation and into adulthood. The combination of virus and gut fungi in the same lentic system may enable vertical viral transmission into adult mosquitoes.

An example of this type of gut viral transfer, albeit in the adult stage, was an epidemic of Rift Valley fever virus in a part of Africa where it does not normally occur. 
Culex pipiens was not able to transmit Rift Valley fever virus unless also infected by microfilariae (Spielman et al. 2001). After adult mosquitoes blood fed, microfilariae entered and then pierced the gut lining, allowing viral transmission into the hemolymph and eventually into the saliva for disease transfer (Spielman et al. 2001). In my study, some extruded gut fungi spores began forming thin elongations beyond the anchor point on the outside of the peritrophic matrix (Plate 1.3). These were actively growing and responding, presumably to host cues, possibly extending further if given more time. Alternatively, these thalli could play a role in the yet to be described adult ovarian cyst stage, as well as assist with viral transfer into larvae/adult mosquitoes, similar to how microfilariae create holes.

Surveys are an important first step to understanding the dynamics of gut fungi biology whether in man-made or pristine underexplored areas. Gut fungi biogeography, seasonal variation, and host distributions are beginning to be understood as these relate to Idaho mosquitoes. Further explorations that also consider midges and other related host families (see chapter 2), ovarian cysts in later development stages, and developing molecular approaches for environmental sampling or assessing possible viral presence in transfer from larva to adult, would take these obligate endosymbionts to the next levels of research. 


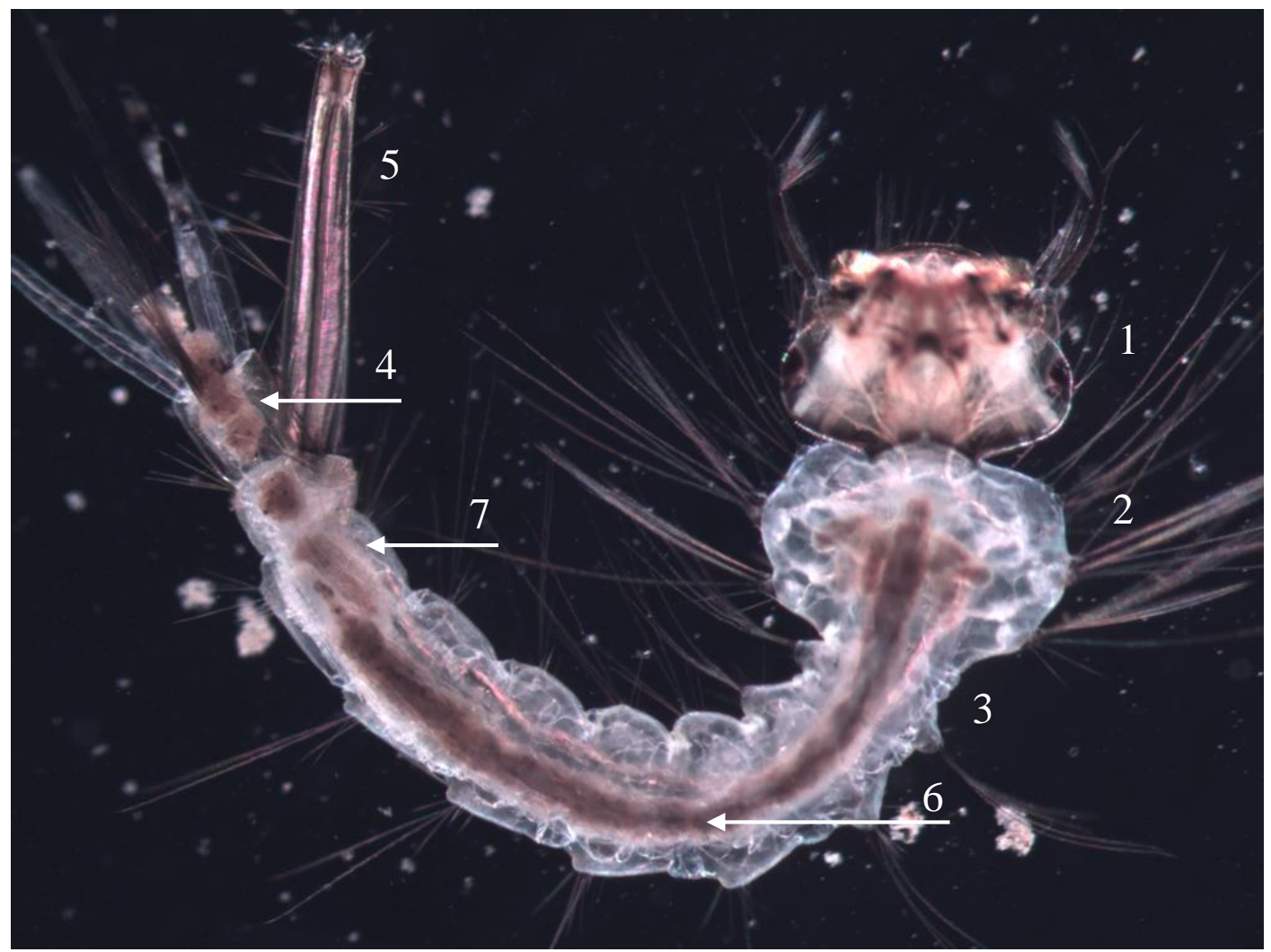

Plate 1.1: Culex sp. mosquito larva, $4^{\text {th }}$ instar. The gut and food bolus can be seen through the cuticle. Anatomical regions labeled as: 1) Head. 2) Thorax. 3) Abdomen. 4) Saddle and anal segment. 5) Siphon. 6) Midgut. 7) Hindgut. 


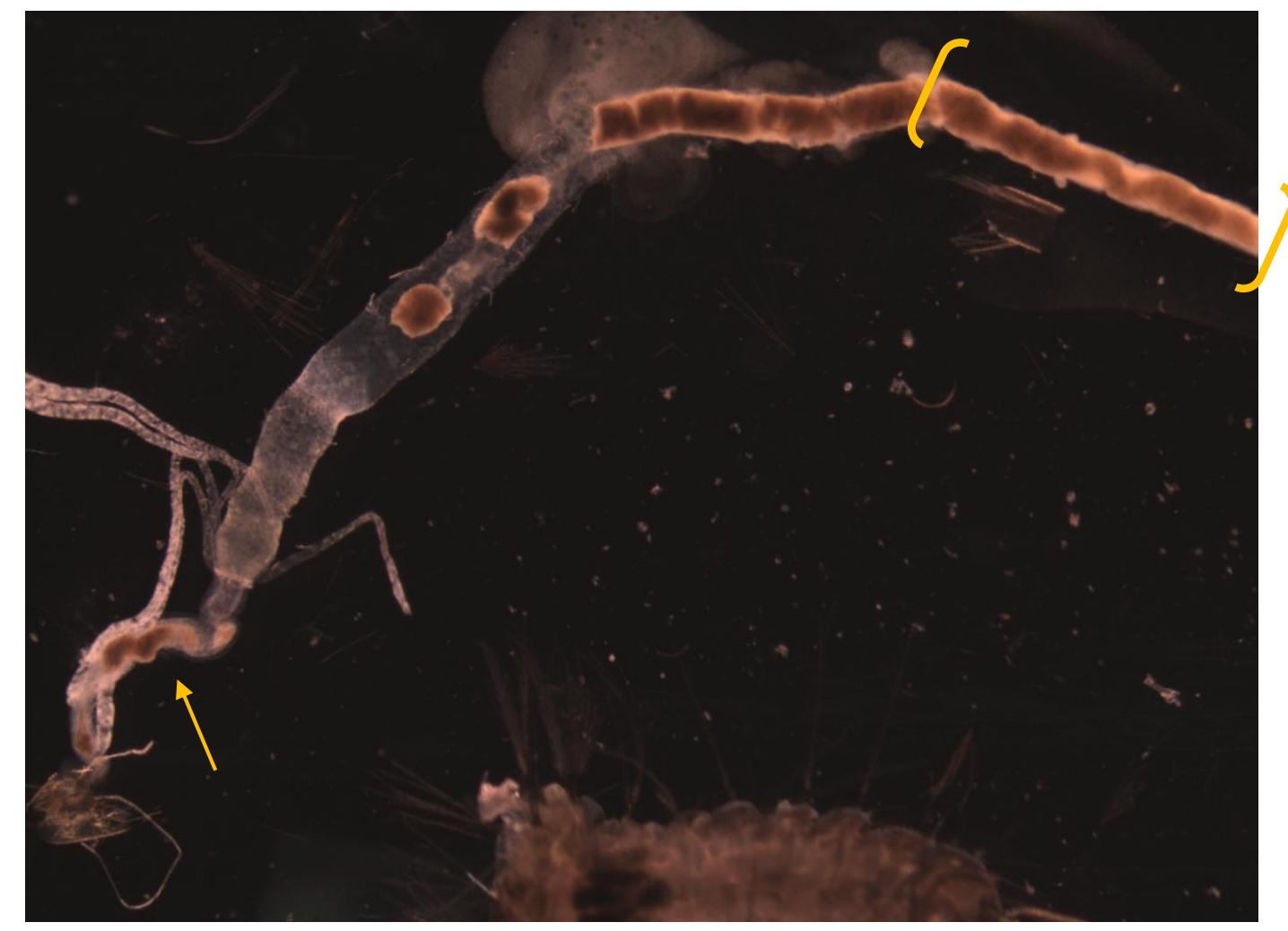

Plate 1.2. Example of larval mosquito digestive tract. Arrow indicates hindgut, where mature gut fungi dwell. Brackets encompass the midgut, which having just slid out from inside the epithelial cells (left) contains the food bolus. 


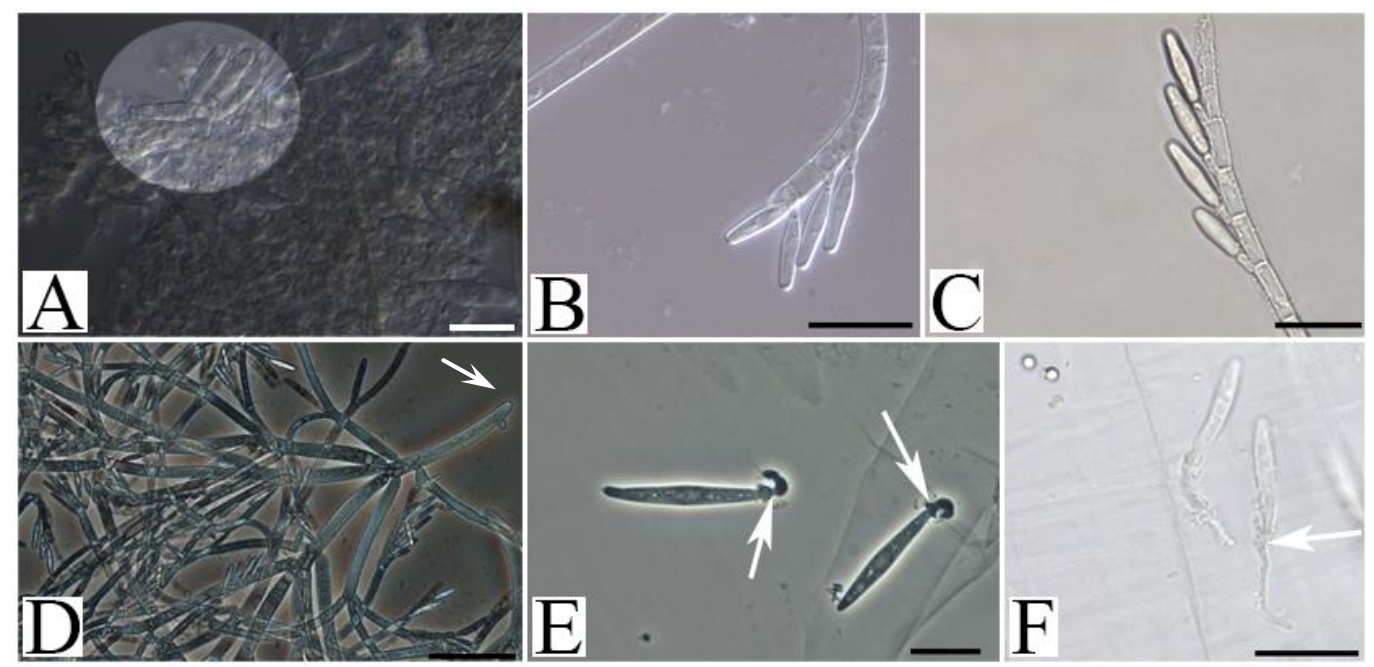

Plate 1.3: Examples of all fungi collected. A. Smittium minutisporum spores mixed with detritus from hindgut, medially swollen (NIC $\left.{ }^{*}\right)$; (Bar $\left.=25 \mu \mathrm{m}\right)$. B. Zancudomyces culisetae trichospores attached to generative cells $\left(\mathrm{NIC}^{*}\right) ;(\mathrm{Bar}=20 \mu \mathrm{m})$. C. Smittium culicis trichospores attached to generative cells with $\left(\mathrm{NIC}^{*}\right)$; $($ Bar $=25 \mu \mathrm{m})$. D. Zancudomyces culisetae with simple holdfast indicated by arrow; (Bar $=50 \mu \mathrm{m})$. E. Arrows indicating pierced midgut (peritrophic protein matrix) of a Culex tarsalis by precociously extruded spores of $S$. culicis, which produced a hook-like holdfast; (Bar $=20 \mu \mathrm{m}$ ). F. Pierced midgut (arrow) of Culiseta alaskaensis via extruded spore of $Z$. culisetae; spore appearing to elongate further than just an anchoring hold fast (NIC*); $($ Bar $=20 \mu \mathrm{m})$. *Nomarski Interference Contrast 


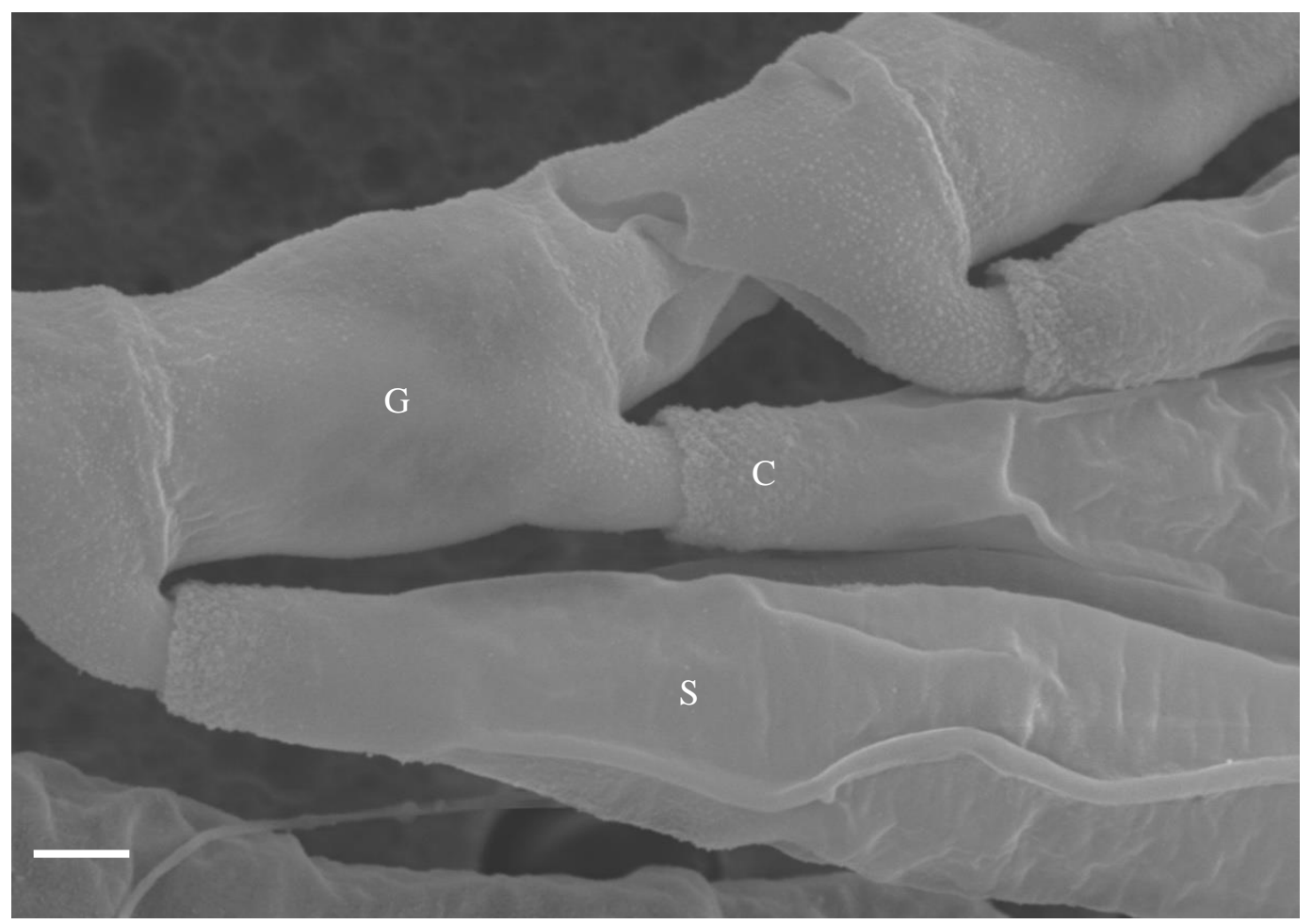

Plate 1.4: Zancudomyces culisetae morphological structures in the scanning electron microscope, bar $=1 \mu \mathrm{m} . \mathrm{C}=$ collar region, $\mathbf{G}=$ generative cell, $\mathrm{S}=$ trichospore attached to generative cell. 


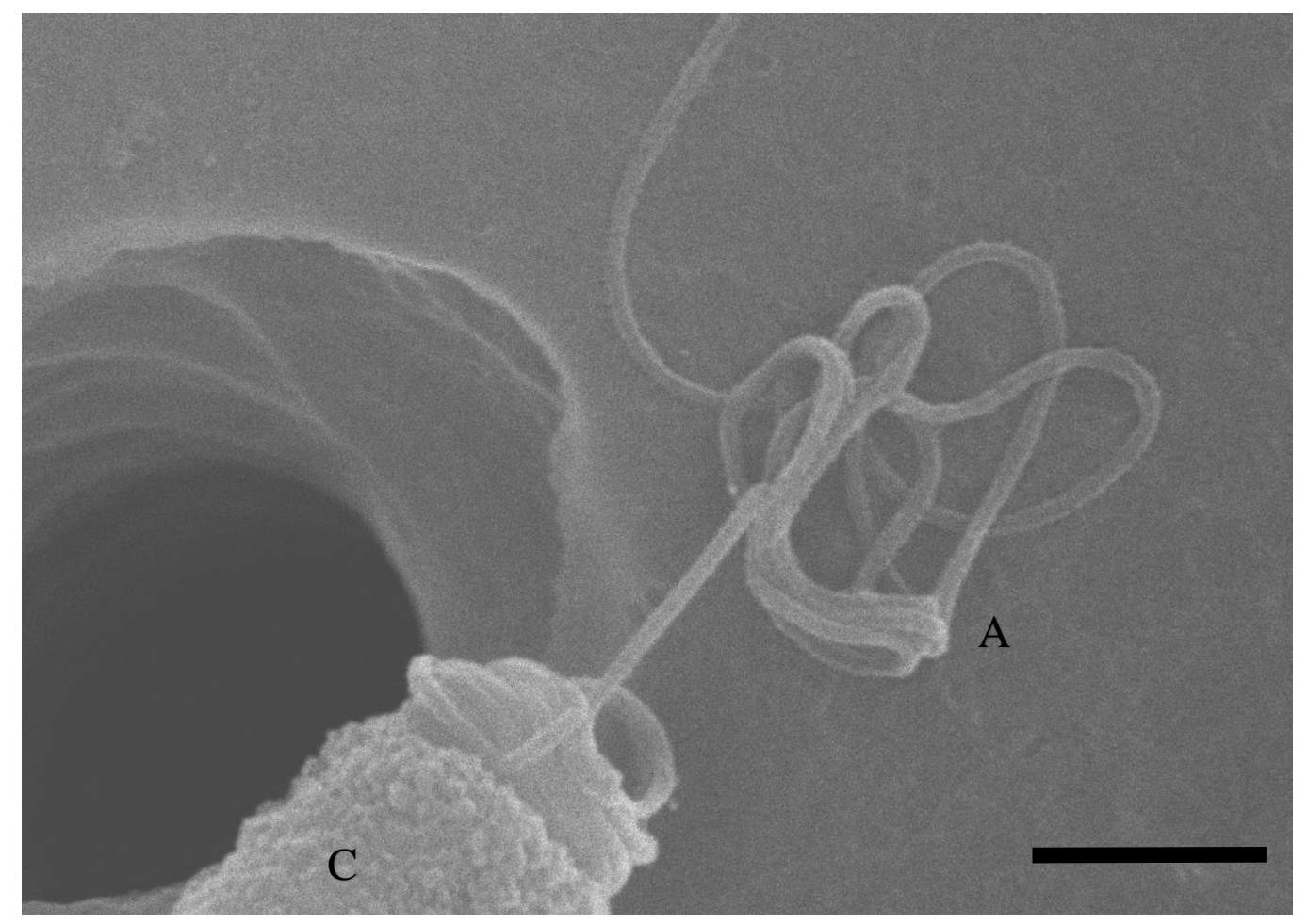

Plate 1.5: Zancudomyces culisetae morphological structures in the scanning electron microscope, bar $=1 \mu \mathrm{m}$. Proximal end of detached trichospore. Nonmotile appendage (A) still coiled and unwinding from inside spore collar (C). 


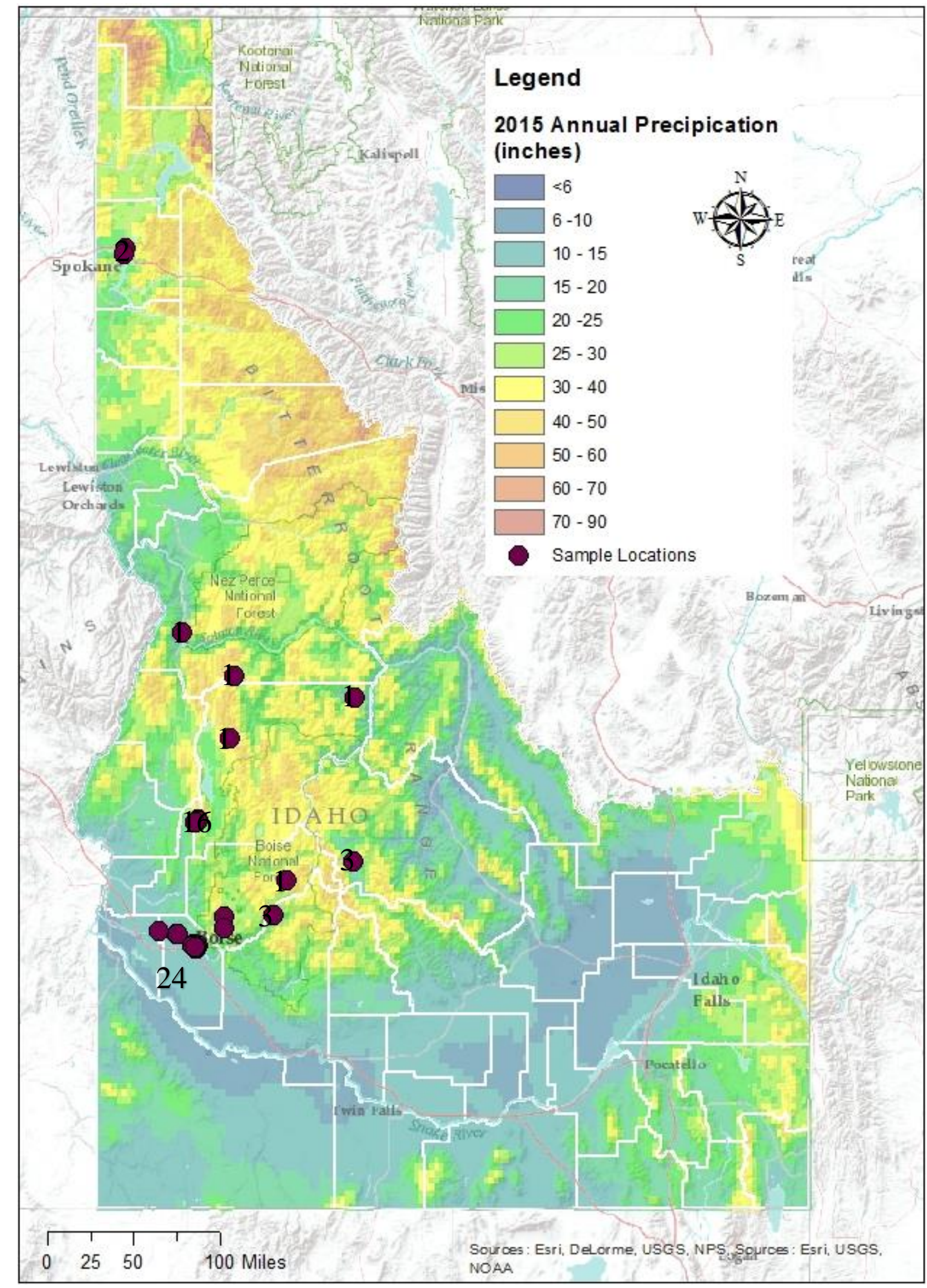

Figure 1.1: Survey sites of mosquitoes in Idaho, USA. Collections $(n=53$, numerical values near sample locations equal the number of collections at those points) plotted with county boundaries, topography, and annual rainfall map for 2015, similar rainfall patterns were observed in 2016. (NACSE, 2017) 


\title{
DISPERSAL ECOLOGY OF A GENERALIST GUT FUNGUS, ZANCUDOMYCES CULISETAE, AN OBLIGATE ENDOBIONT OF AQUATIC DIPTERA IN A MOUNTAIN STREAM ECOSYSTEM
}

\begin{abstract}
The Harpellales are arthropod associated micro fungi that dwell as obligate endosymbionts in the digestive tract of immature aquatic insects. Zancudomyces culisetae is one member of the order of gut fungi, now recognized as an ecological group, but traditionally known as Trichomycetes. This study provides further understanding of the ecology and life history of Z. culisetae as it relates to both lentic and lotic sites associated with a mountain stream ecosystem, Renwyck Creek, including its nearby tributaries and confluences. Headwater reaches of Renwyck Creek were explored for boil, splash, and flood zones and were sampled to better understand the nature of gut fungal spore dispersal and distribution, along with its host range and capacity. Upstream from infested Culicidae habitat, collected Chironomidae and Thaumaleidae were infested with the same gut fungus, therefore providing the opportunity to act as potential fungal reservoirs. When mosquito larvae were collected from puddles filled by lotic systems, infestation was $100 \%$ once they reached $4^{\text {th }}$ instar. This high incidence of infestation suggests that trichospores may, at least, be dependent on the connection between lotic and lentic habitats for trichospore dispersal.
\end{abstract}


$\underline{\text { Key words }}$

Culiseta alaskaensis, Eucorethra underwoodii, Kickxellomycotina, midge, solitary midge, stream ecology, symbiosis 


\section{Introduction}

Harpellales is a globally distributed ecological group of arthropod-associated fungi that reside in the digestive tracts of their hosts (Lichtwardt, 2004). The two families of Harpellales, Legeriomycetaceae and Harpellaceae, are roughly delineated on the basis of whether species have branched thalli and are hindgut versus unbranched midgut dwellers. Hosts of Harpellales include taxa associated with immature stages of dipteran aquatic arthropods, such as mosquitoes (Culicidae) and midges

(Chironomidae/Thaumaleidae). These families of dipterans often harbor gut fungi and have been relatively well investigated compared to some other arthropods (Lichtwardt et al., 1986, 2004, 2007). However, not well studied is the dispersive capacity and cycling of the obligate endosymbiont, Zancudomyces culisetae (Harpellales: Legeriomycetaceae), such as between the mosquito Culiseta alaskaensis (Diptera: Culicidae) and potential reservoir hosts. Zancudomyces culisetae is an ideal candidate to study the dispersal ecology between varied hosts because it colonizes different families of dipteran larvae, including Ceratopogonidae, Chironomidae, Culicidae, and Psychodidae (Lichtwardt, 2007). These varied hosts of $Z$. culisetae inhabit both lotic and lentic systems, increasing its potential dispersive boundaries and capacity as a generalist endosymbiont of stream systems (Lichtwardt, 2007).

Past surveys of gut fungi have been conducted worldwide, focusing mainly on taxonomy, biodiversity, and discovery. Most of these surveys have been conducted on lotic systems, with many fewer in lentic habitats, and none, to the author's knowledge, of the interaction of lotic and lentic systems together (concerning Harpellids) (Lichtwardt et al., 1986, 2004, and 2007). Zancudomyces culisetae has been found in varied lentic sites, 
including discarded bottles, tires, and roadside puddles (Lichtwardt, 2004). Using $Z$. culisetae as my focus, I attempted to explore whether there is a common link, or a dispersive boundary, between lotic and lentic habitats.

In this study, I targeted the specialized aquatic habitat and larvae found in a timberline mountain stream system, Renwyck Creek, Gem Co., Idaho. This system is fed by spring water year-round and has varying microhabitats consistent with the preferences and needs of specialized aquatic larval stages of Diptera, well known as hosts of $Z$. culisetae (personal observation). I began collections in the flooded lentic habitat, seeking Culicidae larvae and adults (Fig. 2.2). More specialized larvae, like the drain fly Psychodidae, dwelt where converging small springs enter the main channel, forming thin films of water called splash zones (Fig. 2.3). Continuing to the source of the stream water, i.e., spring boil zones, I sought Chironomidae larvae (Gillespie et al., 1994; Mackie, 2004). Artificial containers were also placed along Renwyck Creek and used to attempt to collect additional C. alaskaensis and Z. culisetae specimens.

Springs (boil zones) (Fig. 2.4) occur where discharges of groundwater meet the surface and provide a consistent source of water, temperature, and chemistry for its biota (Giller and Malmqvist, 1998). Further down the system, splash zones (Fig. 2.3) form alongside areas of torrential water flow, steep gradients, and large boulders. The biota here are specialized and able to tolerate high carbon dioxide levels (Giller and Malmqvist, 1998). Predictable seasonal variation of water levels in the flood zones of lotic systems provided unique opportunities for food and habitat for invertebrates (Fig. 2.2) (Statzner and Borchardt, 1994). When considering where to hunt for specialized hosts in mountainous stream systems, key factors considered include oxygen, seasonal 
flow, and substrate (Giller and Malmqvist, 1998; Mackie, 2004). As much as could be gauged (personal observation), habitat boundaries and all sources of water in the field were interconnected tributaries flowing into Renwyck Creek, offering a productive setting for collecting and exploring the symbiotic relationship of $Z$. culisetae amongst its potential hosts.

The mountainous area surrounding Renwyck Creek is considered semi-arid, as $60 \%$ of moisture occurs in the form of snow during the winter months, leaving observed lentic aquatic habitats in the drier summer months connected to stream systems (Kottek et al., 2006). I hypothesized that various families of dipterans would be found in specialized zones along Renwyck Creek, and would be infested with Z. culisetae, thus acting as potential reservoir hosts for the fungal populations dispersing to lentic sites downstream. Since the aquatic spores (asexual or sexual) of harpellids are non-motile, the infested specialized lotic hosts would need to reside upstream of or be concurrent with mosquito larvae to act as fungal reservoirs. Mosquito larvae habitat changes seasonally, and due to the randomized locations of lentic sites, the limits in dry soil that trichospores can endure (five months) are reached (Williams, 1983). This necessitates the need for fresh inoculation of gut fungi from upstream sources. In chapter 1, I hypothesized that isolated site dispersal of $Z$. culisetae could occur via a putative ovarian cyst stage in flying adult female mosquitoes (Culicidae). To investigate the possibility of vertical transmission, I dissected naturally occurring adult female mosquitoes collected near Renwyck Creek.

Morpho-taxonomy is the traditional approach for species identification. Therefore, wild-caught (wild-type) trichospores of Z. culisetae from Renwyck Creek were scrutinized for their consistency in size and undertaken with collection ID-333. 
Vouchers for wild-caught fungal collections were compared with those from axenic isolates of Z. culisetae to assess possible variations in spore size as observed from previous 2015 collections.

\section{Materials and Methods}

\section{Collection Location}

The study was conducted from June through November 2016, at several sites along Renwyck Creek, its tributaries, and the merging confluence zone of Renwyck Creek and Squaw Creek (Gem Co., ID, USA (Fig. 2.1, appendix: Table 2.1). Immature aquatic stages of dipterans were collected in mountainous and forested stream habitats. Collection locations spanned approximately 3.2 kilometers $(\mathrm{km})$ and 148 meters $(\mathrm{m})$ of elevation change along Renwyck Creek.

Stream habitats or zones, expected to harbor known host families of Z. culisetae, were targeted for collection. Specifically, boil zones, splash zones, and flood areas. The beginning of a small spring, called the boil zone, where water bubbles up and forms slow flowing systems, were sought for larval Chironomidae (Fig. 2.4). Hygropetric habitats and splash zones were found next and formed where the stream became steeper and wider ( 1 m) (Fig. 2.3). The faster flow formed thin films of water on boulders, which were scoured for Psychodidae. Further downstream, Renwyck Creek widened $(<10 \mathrm{~m})$ as the terrain flattened out, leaving lentic puddles randomly strewn along and eventually just beyond the bank, where Culicidae were targeted for collection (Fig. 2.2). Any other nontarget hosts found in these areas were also gathered, dissected, and examined for gut fungi. 


\section{Collection Technique}

At each aquatic collection site, samples of insect larvae were gathered with a unique, unused fine mesh fish net and placed into a new one-gallon plastic storage bag. Water, leaves, debris, and some detritus from the collection site were added to the bag to offer larvae a relatively natural habitat and familiar food source. Samples were placed in a cooler with ice for transport to the laboratory, then stored at $4{ }^{\circ} \mathrm{C}$ until dissection of larvae. Dissection occurred within seven days of collection. Sterile plastic transfer pipets were labeled with a collection number and used only with that collection to prevent cross contamination among samples. Four of the longer-lasting lentic sites were sampled up to 4 times during the season (sites: 1, 7, 8, and 10) (appendix: Table 2.1).

\section{$\underline{\text { Artificial Containers }}$}

On June $2^{\text {nd }}, 2016,15$ clear artificial containers (Manufacture \#4SFSCW-4, Recycle \#7 other, Cambro, Huntington Beach, CA) were buried within $10 \mathrm{~cm}$ of ground level in shady areas of moist soil $\sim 1 \mathrm{~m}$ from the water's edge of Renwyck Creek. The containers, which were evenly distributed along a $1.6 \mathrm{~km}$ stretch of Renwyck Creek ( 0.8 $\mathrm{km}$ apart), were each filled with $1.5 \mathrm{~L}$ of creek water and a $1 \mathrm{~cm}$ deep layer of detritus and fine silt collected from the nearest edge of the creek. The detritus and fine silt added to the containers were intended to approximate conditions in naturally occurring puddles left by Renwyck Creek after high flow. The artificial containers were covered with 6.3 $\mathrm{mm}$ mesh wire in an attempt to prevent animals from tampering with the water and its contents. Artificial containers were checked every three weeks, and $4^{\text {th }}$ instar mosquito larvae were collected when present. The containers were retrieved on October $4^{\text {th }}, 2016$. 


\section{Laboratory Handling, Processing, and Vouchering}

Axenic culture isolation attempts were made for any Harpellales, where sufficient material allowed, per techniques described by Lichwardt et al. (1986, 2004). Some of each axenic culture was stored in vials of cryopreservative (PL.170, Prolab Diagnostics' Microbank) at $-80^{\circ} \mathrm{C}$ in a New Brunswick Scientific Ultra Low Temperature Freezer (Model U535 Innova) for future use. Cryopreservative represents a new approach for axenic culture isolation with these fungi. Each sample was labeled with date, temperature, collection location, and given a collection identification number (appendix: Table 2.1). Each collecting site was given a unique number. Each collection event has its own collection code, but the site number remained the same if it was a subsequent revisit. When needed, Z. culisetae (ID-333) was grown in BHI culture after storage in previously described cryopreservative. The last culture grown had been stored for 8 months and successfully revived.

\section{Host Identification}

Larvae were identified using the regional identification key in Brothers (2003). Some larvae and adults (hatched in vitro) from each collection were stored in $70 \%$ ethanol as voucher specimens (Lichtwardt et al., 1986, 2004). Vouchers have been deposited in the trichomycete laboratory of Dr. Merlin White at Boise State University. Fungal Identification

Mosquito larvae dissected were $4^{\text {th }}$ instar, as they are larger and have a greater potential, with longer intermolt durations, for fungal infestation (personal observation). After placing the larva on a microscope slide in a drop of sterile $\mathrm{diH}_{2} \mathrm{O}$, the head was removed with sterile sharpened forceps. The saddle was then removed, leaving the siphon 
attached but removing the hindgut and midgut (Plate 1.1,1.2). The hindgut and midgut were separated at the pyloric sphincter. The hindgut was transferred to a fresh drop of sterile $\mathrm{diH}_{2} \mathrm{O}$ on a new microscope slide. The midgut was given a minute to allow the food bolus and epithelial layer to separate via osmotic pressure, leaving behind the peritrophic protein matrix. The clear peritrophic matrix was then placed into a fresh $\mathrm{diH}_{2} \mathrm{O}$ drop with the hindgut. The hindgut was then lacerated with forceps. A glass cover slip was placed over the fresh $\mathrm{diH}_{2} \mathrm{O}$ drop, gently using capillary action of the $\mathrm{diH}_{2} \mathrm{O}$ to eliminate air bubbles. The entire area of the cover slip was reviewed in a linear back and forth pattern at 200x magnification using a Nikon Eclipse 80i microscope. If harpellid gut fungi were detected, the slide was labeled with the date, collection number, initials, serially increasing code, and preserved in lactophenol cotton blue (LCB). The glass cover slip was double sealed with Revlon brand nail enamel (clear 771), (Lichtwardt et al., 1986, 2004); (Bench and White 2012).

Released, free floating trichospores of $Z$. culisetae were collected from the larval hindgut and measured for length, width, and collar size (Plate 1.3). Averages of each measurement were then compared to morphometric data of known samples and used to delineate between known species. Taken together, spore length, how wide and where on the spore was the widest (i.e. medial), and features of the collar (where the spore was attached to generative cell) shape and size, are the characteristics used for identification purposes (Lichtwardt, 2007). Trichospores were imaged and measured with Spot Advanced software (4.6) and a 2 MP Spot Color Mosaic camera (Diagnostic Instruments, Sterling Heights, Michigan). 
Axenic culture attempts were made using the protocol of Lichtwardt (2007, chapter 3) and are stored in the trichomycete laboratory of Dr. Merlin White at Boise State University. If there were multiple clumps (with 3-20 spores each) of gut fungal thalli available, one was used for glass slide preservations and one or more for culturing. Voucher specimens were always taken first, and then the rest was used for culturing attempts. Axenic cultures are important as they provide a means for future genetic analysis and in vitro laboratory experimentation.

\section{Spore Volume Comparison}

To analyze differences in trichospore size between wild-type and culture-type spores of Z. culisetae (ID-333), the volume of detached, non-extruded, spores were compared using a density plot made in R Studio and analyzed with a two-sample Kolmogorov-Smirnov test. (Fig. 2.5). Larvae stored at $10^{\circ} \mathrm{C}$, from collection ID-333, were dissected within 1 day of collection for best representation of wild-type spore volumes. Cultured spores used in the density plot were grown in BHI agar, with sterile water overlay, at $10^{\circ} \mathrm{C}$ for 14 days (Lichtwardt et al., 1986, 2004, chapter 3). Spore size was measured using the length and width of asexual trichospores. Based on the shape of an ellipse, trichospore volume was estimated using the formula:

$\mathrm{V}=(4 / 3 \pi)($ length $) \cdot 2$ (width)

\section{Results}

Renwyck Creek/Second Fork Squaw Creek and its confluences provided ample yields of aquatic arthropods for examination. The main surge of winter run-off/snowmelt had slowed by June 2016 and puddles began forming below the previous high-water marks. Mosquito (Culicidae) larvae first became available for collecting when $3^{\text {rd }}$ instar 
was reached in early July (Table 2.1). Dissection of adult mosquitoes yielded no evidence of ovarian cyst stages. Other non-target aquatic dipteran larvae were found, noted, and collected for later dissection, including Eucorethra underwoodi (Chaoboridae: sites 3, 7, 14, 15), Chironomus riparis (Chironomidae: site 10), Dixidae (site 13), Stratiomyidae (site 12), and Thaumaleidae (site 4) (Table 2.1). Each family was associated with a particular zone and along varying parts of the aquatic system. Chironomidae and Stratiomyidae were in the boil zone of an emerging spring that fed into Renwyck Creek (site 12) (Fig. 2.4). Thaumaleidae were found in a steep section further downstream among the splash zones of a boulder-strewn, fast-flowing current (Fig. 2.3). Culicidae and Chaoboridae were collected in the lower, flattened and flooded areas of both creeks, which left puddles along the bank edges (Fig. 2.2) (appendix: Table 2.1). No Psychodidae were identified from the splash zone collections.

Zancudomyces culisetae was found in Chironomidae, Culicidae, and Thaumaleidae. The predacious Chaoboridae (Eucorethra underwoodii) did not yield any gut fungi (N=76). Only one species of mosquito was collected, Culisetaalaskaensis, but it harbored Z. culisetae with a $100 \%$ infestation rate. This mosquito was the most abundant host insect collected $(\mathrm{N}=288)$. All examples of putative reservoir hosts were collected upstream of infested mosquito larvae habitat.

Zancudomyces culisetae trichospores were sub-medially swollen with a cylindrical collar. Quantitatively, it had an average asexual spore length $( \pm \mathrm{SE})$ of $16 \pm$ $0.1 \mu \mathrm{m}(\mathrm{n}=97$, range $=13.5-18.8 \mu \mathrm{m})$, average spore width $( \pm \mathrm{SE})$ of $3.8 \pm 0.05 \mu \mathrm{m}$ $(n=97$, range $=2.8-4.9 \mu \mathrm{m})$, and average collar size $( \pm S E)$ of $1.5 \pm 0.04 \mu \mathrm{m}(\mathrm{n}=97$, range $=0.8-2.6 \mu \mathrm{m}$ ) 
Despite careful planning and deployment of artificial containers, including the use of $6.3 \mathrm{~mm}$ galvanized wire mesh coverings, Ascaphus montanus (Ascaphidae) tadpoles were abundant in the artificial containers, except for two which aquatic dipteran larvae were collected (site $10 \& 13$ ). The tadpoles presumably consumed any dipteran eggs that may have been deposited in the containers. Zancudomyces culisetae was not found in the only artificial container to harbor C. alaskaensis larvae (site 10) (Fig. 2.1). However, another gut fungus, Stachylina sp., was recovered from the midgut of Chironomus riparius, cohabiting that same container. No gut fungi were recovered from a non-target host family, Dixidae, collected from artificial container ( site 13).

Spore volume of wild-type spores had the greatest density of spores at $\sim 1000 \mu \mathrm{m}^{3}$ and was less than the spore volume of cultured spores which had the most clustering at about $\sim 1600 \mu \mathrm{m}^{3}$ (n=42 wild-type, $\mathrm{n}=42$ culture-type, $\mathrm{P}<0.001$ ), (Fig. 2.5).

\section{Discussion}

Many previous studies on gut fungi have been a mix of field surveys and some local, long-term collections that have helped to establish axenic cultures and species level morphometric data (Lichtwardt, 2007). These cultures allowed for in vitro laboratory experiments and helped to ask and answer questions about their relationships, physiology, host specificity, and even trichospore longevity (Williams, 1983; Moss, 1998; Misra and Horn, 2001; Lichtwardt et al., 2004). Although surveys have revealed a worldwide distribution of gut fungi, much of the globe remains unsampled (Lichtwardt et al., 2004). Species of Harpellales vary in their known patterns, but Z. culisetae is considered widespread and cosmopolitan (Lichtwardt, 2007). 
Along Renwyck Creek and its confluences, Z. culisetae would be recognized and considered as a generalist that infests multiple dipteran families of aquatic arthropod larvae (Lichtwardt, 2007). In many ways harpellids are still studied with a discoverybased approach, owing to the multitude of known arthropod taxa that have yet to be collected and dissected for their potential gut fungal endosymbionts (Lichtwardt et al., 2004). Herein, the list of $Z$. culisetae hosts expands to include a first report of infested Thaumaleidae larvae, which was collected in the steeper, narrower section of Renwyck Creek's splash zones.

Culiseta alaskaensis (Culicidae) overwinter as adult females and in early summer lay egg rafts along stream systems (Becker et al., 2003; Carpenter and LaCesse, 1955). This behavior is an important aspect of its life history as it relates to Z. culisetae. The host depends on the consistent or inconsistent nature of annual winter runoff in flowing systems to leave behind suitable lentic habitat for larvae. Due to the seasonal change of puddle locations, gut fungi must also have a reliable way of inoculating their most abundant host, the mosquito. The spring, as well as splash zones of the stream system, both yielded hosts infested with $Z$. culisetae. These specialized zones occurred in the confluences merging into the slower, wider section of the stream system where suitable mosquito larvae habitat can be found (Fig. 2.2).

This finding supports the prediction that reservoir host species for gut fungi are present in this permanent system, and upstream of seasonal puddles. Having the ability to connect between the lotic and lentic systems through seasonal water level changes, these non-mosquito hosts have the potential to supply Renwyck Creek puddles with viable spores of $Z$. culisetae. These reservoir hosts are an important addition to the knowledge 
of the life history of Z. culisetae and add to trichospore dispersal mechanisms a more complex and intertwined system amongst varied hosts, than previously realized.

The lentic puddles of Renwyck Creek provide a suitable habitat for both mosquito larvae and gut fungi in two ways. First, water is fed consistently from the nearby stream, through both infiltration of the silt directly and into puddles, thereby keeping them from drying out in summer heat. Second, the mosquito larvae have the means to become inoculated with spores and find food as puddles are formed. The spring high water floods from the stream, carves out a depression (Fig. 2.2), and deposits an inoculum of spores amongst the left behind detritus and silt. Once the mosquito larvae are established in great quantities in one of these more contained lentic areas, they would essentially produce large amounts of fungal spores for cycling the fungus further in the puddle and for later distribution downstream.

Renwyck Creek is an ideal setting for specialized habitat supportive of fungal reservoir hosts and for emerging mosquito habitat. Its aggregate of separated habitats yielded insight into the dispersive ecology of Z. culisetae among multiple dipteran families. Sites such as this help to provide understanding into where spores have potential "over-wintering grounds," as well as how lotic water columns connect to lentic puddles for a more complete life-history of Z. culisetae.

\section{$\underline{\text { Adult Ovarian Cysts }}$}

It has been suggested that there may be an ovarian cyst stage in adult female mosquitoes that propagates gut fungal infestation, similar to what occurs in midges (Moss,1998). To date, there is no evidence for the occurrence of an ovarian cyst stage in mosquitoes (Williams, 1983; Misra and Horn, 2001), including adult female $C$. 
alaskaensis collected along the bank of Renwyck Creek and dissected in the laboratory. Presence of Z. culisetae in Chironomidae larvae gathered from the boil zone flowing into Renwyck Creek raises questions as to how this gut fungus reached the top of the stream system. It is possible that Z. culisetae completes part of its lifecycle as cysts in midge ovaries, thereby allowing for vertical transmission from one site to the next. Many Smittium spp. often associate with mosquitoes and midges in the larval stage (Lichtwardt et al., 2004). Moss (1998) provided evidence of fungal chlamydospores [cysts] of a Smittium sp. on oviposited eggs of a Chironomus sp. (Chironomidae). These Smittium sp. cysts described by Moss (1998) may provide the inoculum for mosquito larvae in newly formed puddles. It should be noted that Moss' Smittium may have been Z. culisetae, as it was originally classified as a species of Smittium based on morphology and was recently revised taxonomically (Wang et al., 2013). The possibility that midges could serve as vertical vectors of $Z$. culisetae should therefore be pursued in future research.

\section{$\underline{\text { Artificial Containers }}$}

The artificial containers placed along Renwyck Creek perhaps raised more questions than answers regarding spore dispersal mechanisms. Mosquito larvae were successfully captured in these containers $(\mathrm{N}=25)$, although they were void of gut fungi (site 10). However, within the same container, Chironomus riparus did show gut fungal infestation with a Stachylina sp. attached to the midgut $(\mathrm{N}=5)$. This finding suggests that either there is an adult cyst stage in Chironomidae that transfers Harpellales from one lentic site to the next, or that silt deposits from the creek contain the spores of this species for inoculation. Chironomus riparus is adapted to low oxygen, silty environments (Giller and Malmqvist, 1998), and so both options are plausible. The small volume (1.5 L) of the 
artificial containers yielded few hosts compared to Renwyck Creek puddles, which often had many more hundreds of mosquito larvae than were present in artificial container site 10 (Fig. 2.1). It is possible, perhaps likely, that larvae in the puddles came from a multitude of adults, which may also increase the likelihood of successful vertical transmission occurring. Although, one artificial container is not enough to draw a conclusion regarding the effectiveness of adult and/or creek inoculation of lentic sites. However, the proof of concept for using this supplemental habitat was upheld and warrants future efforts to develop artificial containers that can house many multiples of hosts and to completely keep frogs or other predators out.

\section{Volume of Spores}

The spore size differences found in Z. culisetae were dependent on whether spores were wild-type or cultured, and were consistent with previous personal observations of spore-size plasticity. For example, comparing mosquito and black fly hosts, trichospores removed from Aedes aegypti (Culicidae) larvae are consistently larger than the same species of asexual spores removed from Simulium vittatum (Simuliidae) larvae reared under similar conditions (Vojvodic and McCreadie, 2009). Whether spore size alone provides a means for increased spore success or fitness is currently unknown. However, when obtaining natural spore size for morphometric analyses, it is important to only use fresh, wild-type spores as spore volume/size appears to be dependent on resource availability. Kim and Adler (2007) found that trichospore size of Harpella melusinae was correlated to host size in black flies (Simuliidae). In my experience, culture media provides unlimited resources that shift spores toward a larger volume as compared to the confined food availability in the host gut at any given time. The limits of 
a petri dish are less than completely mimicking the host gut environment, physiology, and hormonal cues that the fungi may respond to. These trichospores may demonstrate a preference for a larger spore size when nutrients and calories are available. Presumably, with no competition and unlimited access to resources, cultured spores could present with an increase in volume and range. Spore volume is dependent on resource availability; however, it is not known whether spore volume affects survivability and success in the colonization of a new host gut.

Renwyck Creek provided further details of the ecology and life history of $Z$. culisetae in lentic and lotic sites found within this mountain stream ecosystem. The 100\% occurrence of gut fungal infestation in C. alaskaensis found in seasonal puddles, spurred the quest and discovery of specialized dipterans acting as reservoir hosts. Artificial containers show the potential to remediate many questions regarding spore transfer, especially if various treatments could be deployed in the field (e.g. sterilized water vs. stream water). Developing modern methods of eDNA may provide further evidence of $Z$. culisetae's presence in the water column connecting these specialized sites and would be the next step to take for future research studies in this area (Ficetola et al., 2008). 


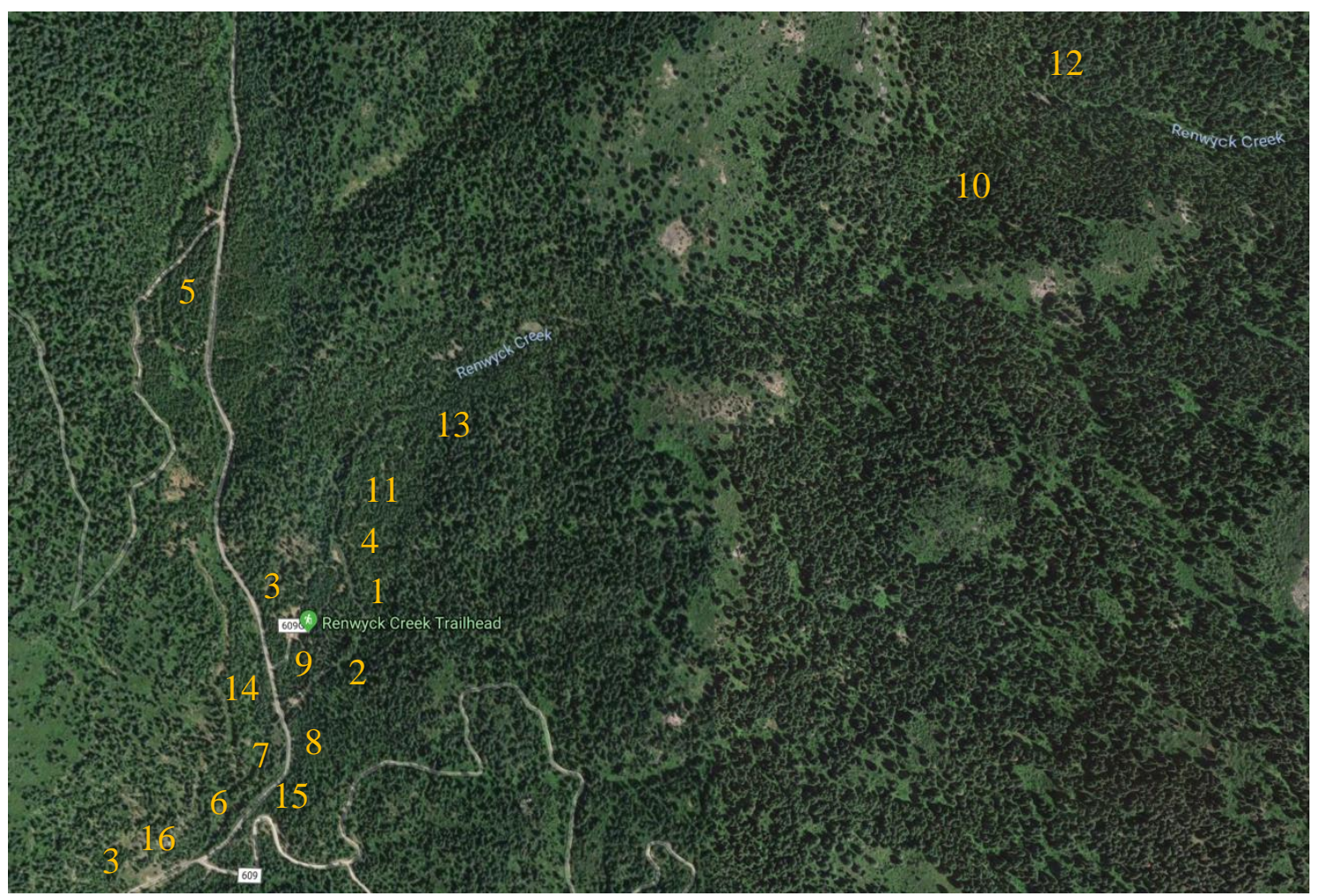

Figure 2.1: $\quad$ Renwyck Creek collections $(\mathrm{n}=23)$ (total sites $=16)$, located $4.8 \mathrm{~km}$ north of Sage Hen Reservoir, Gem Co., ID. Distance covered: $\sim 1.6 \mathrm{~km}$ along a mountainous stream. Sites ranged in elevation from 1425-1573 $\mathrm{m}$ above sea level. Culiseta alaskaensis larvae (Culicidae) were the most abundant Diptera collected $(\mathbf{n}=\mathbf{2 8 8})$. 


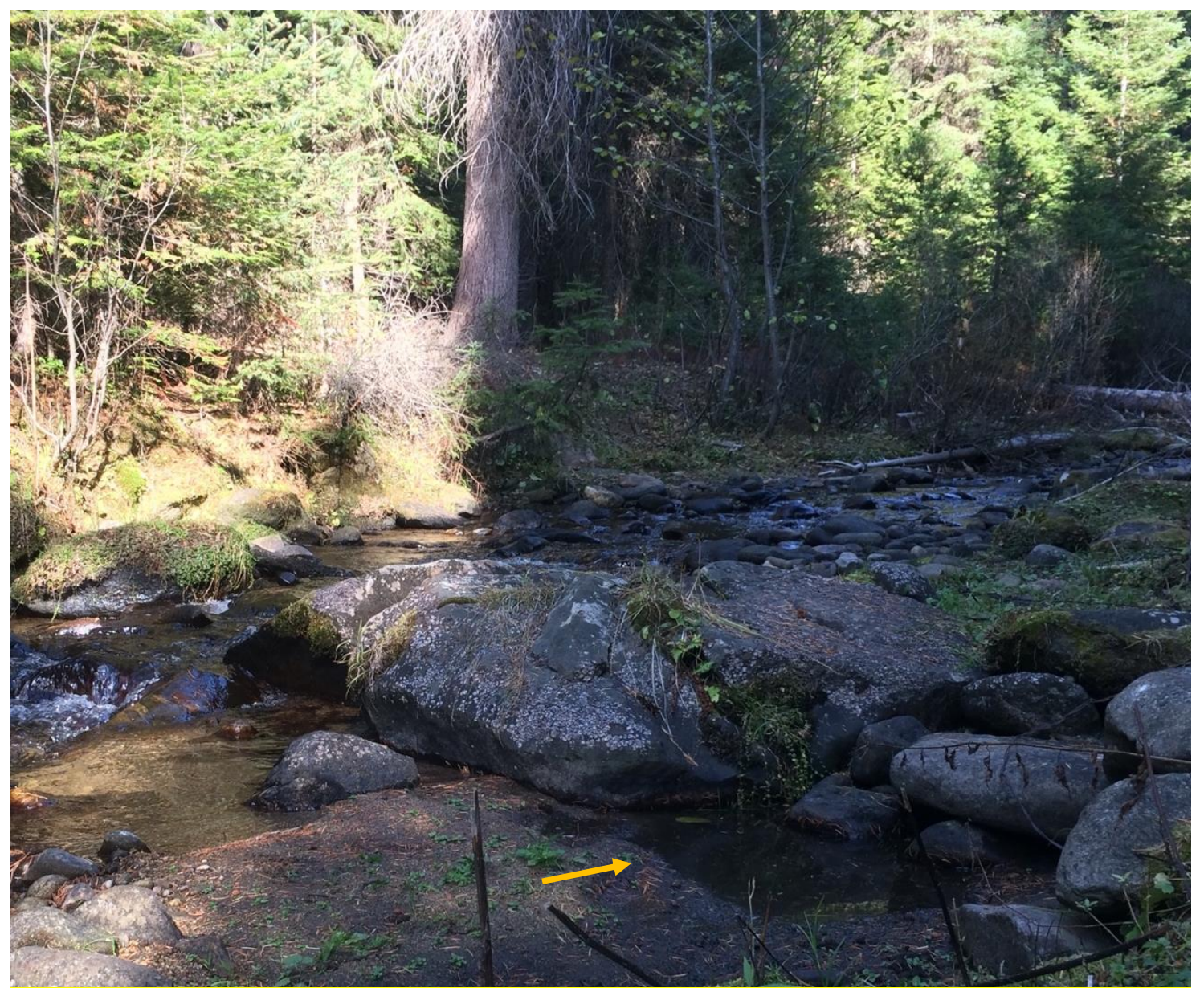

Figure 2.2: Renwyck Creek. Arrow indicates lentic habitat where Culiseta alaskaensis were collected. 


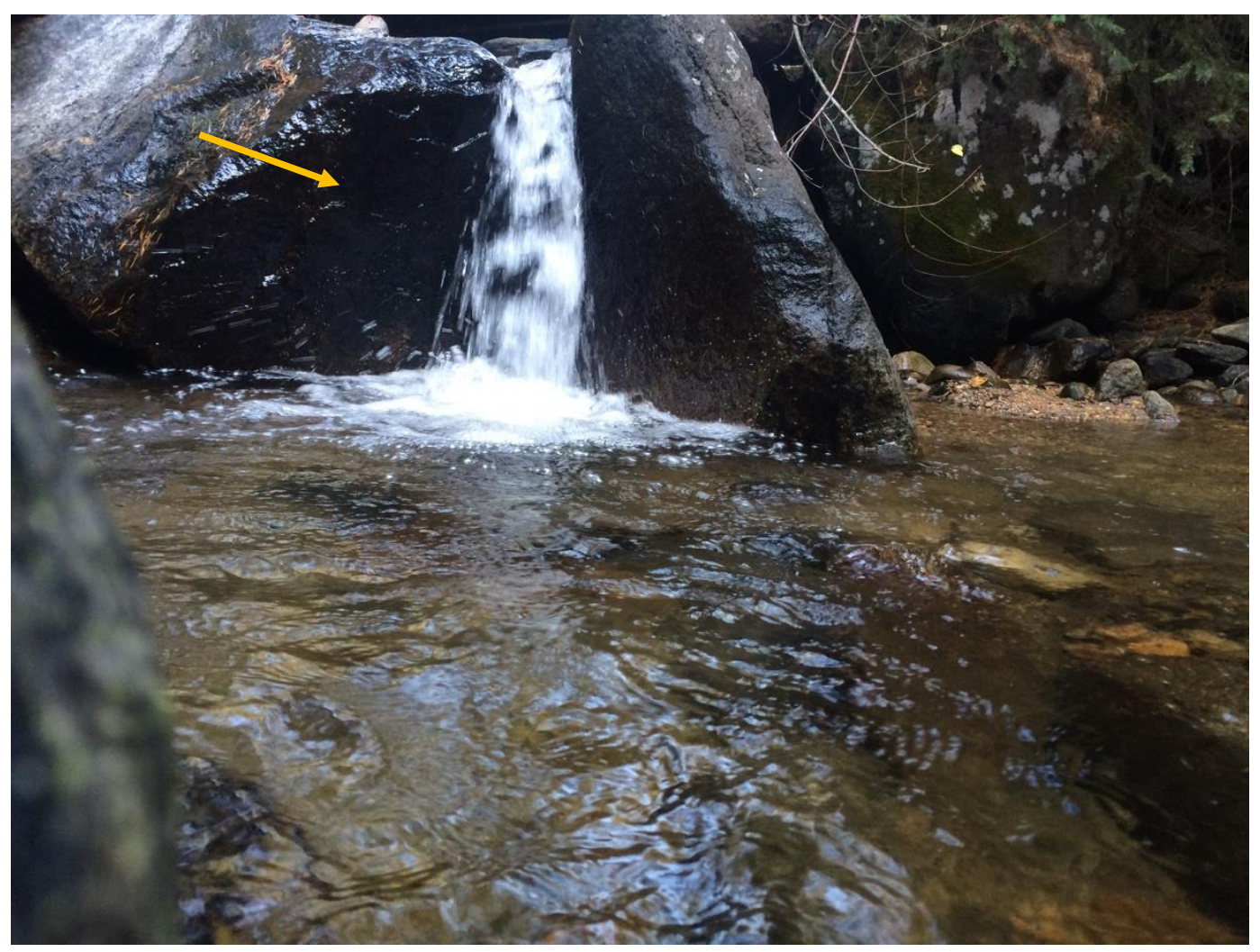

Figure 2.3: Renwyck Creek. Arrow indicates hygropetric and splash zone habitat, where Thaumaleidae larvae were collected. 


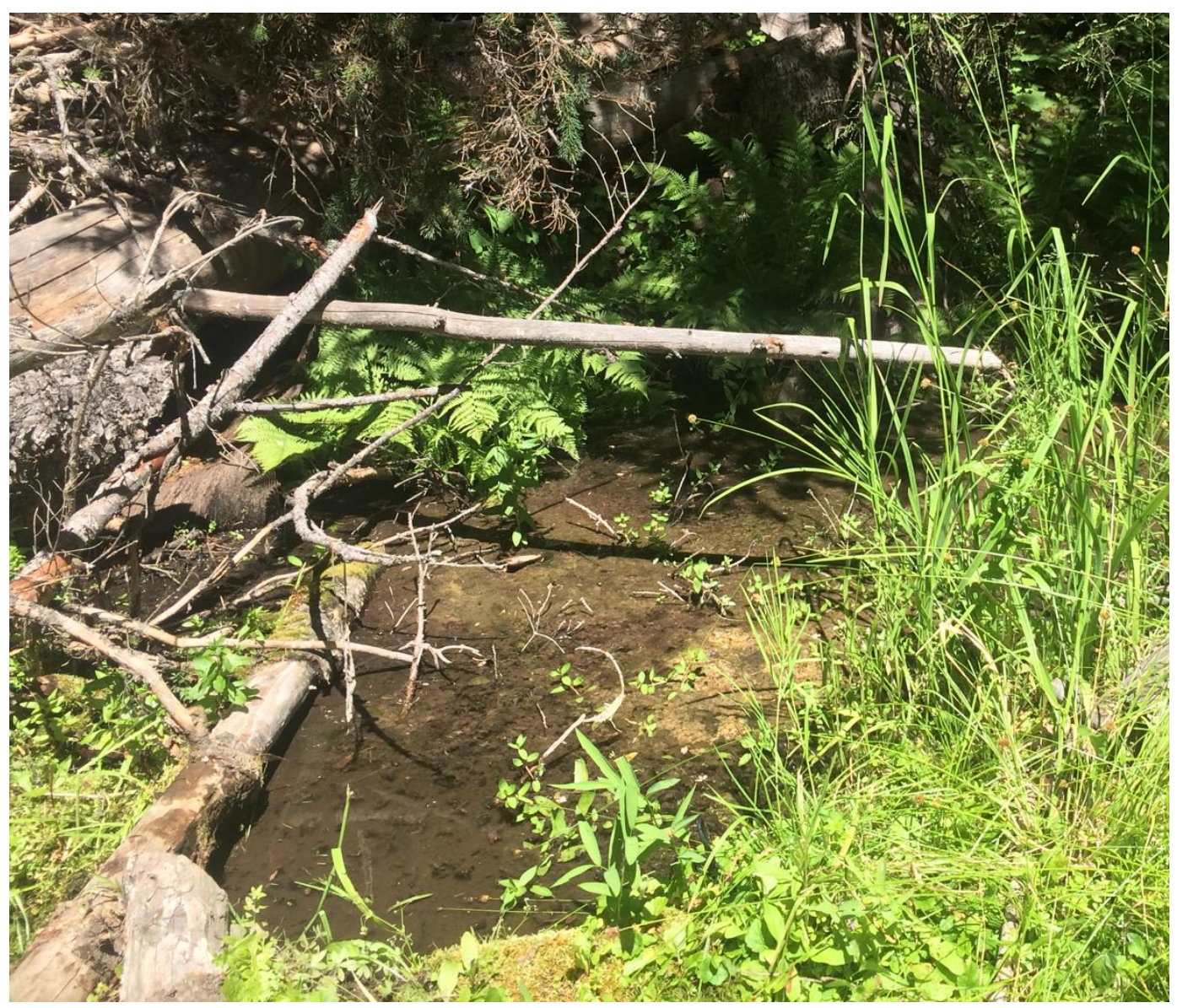

Figure 2.4: Renwyck Creek spring zone. Slow flow, boil zone habitat, where Chironomidae and Stratiomyidae were collected. 


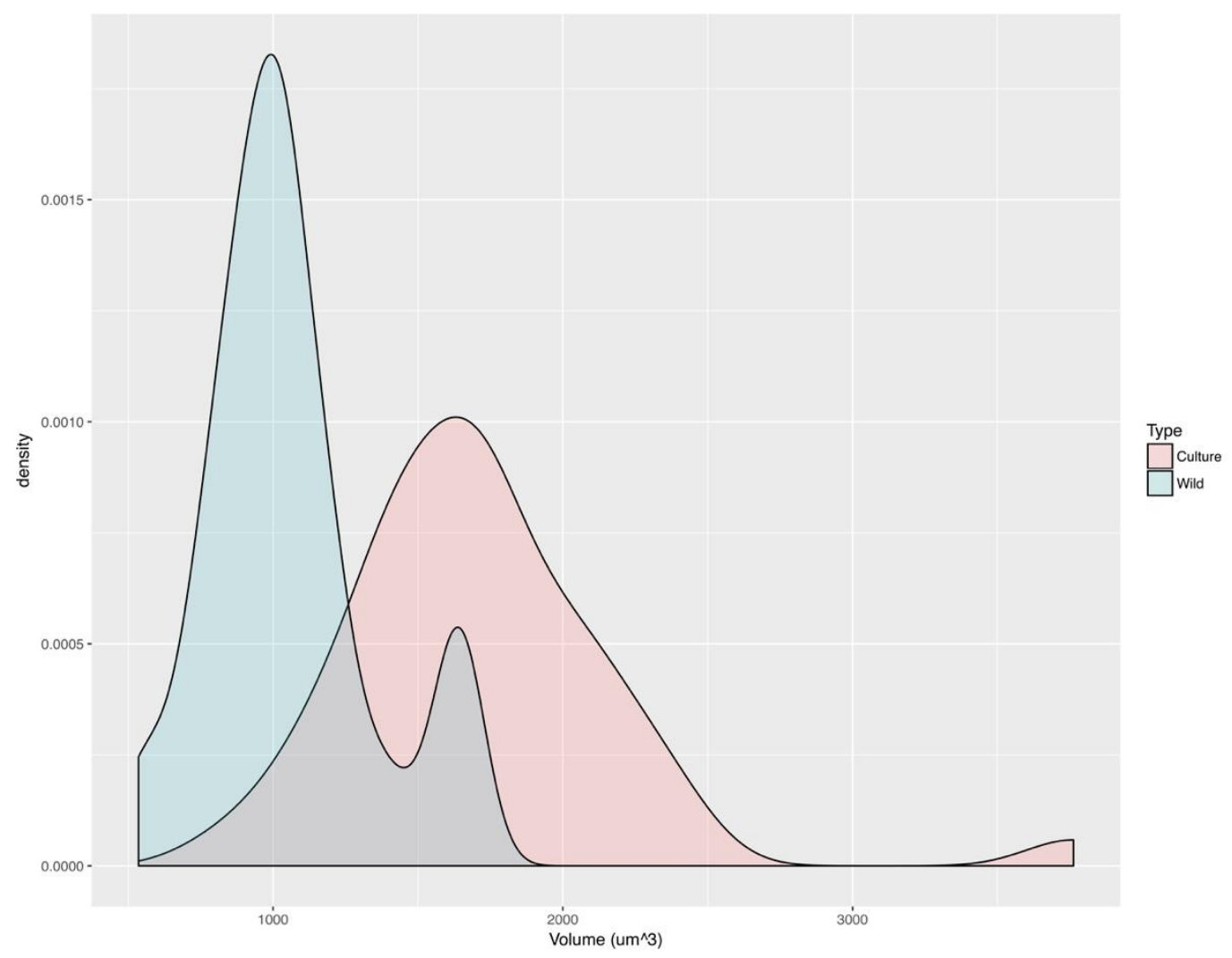

Figure 2.5: A density plot comparison of asexual spore volume of Zancudomyces culisetae of wild type spores $(n=42)$ and cultured type spores $(n=42)$. Spores grown in axenic culture were isolated from the same host species and puddle as the wild type spores (ID-333) and were grown at $10^{\circ} \mathrm{C}$ in $\mathrm{BHI}$ agar with sterile $\mathrm{diH}_{2} \mathrm{O}$ overlay. Wild type spore volume was smaller $\left(\sim 1000 \mu^{3}\right)$ than culture type spore volume $\left(\sim 1600 \mu \mathrm{m}^{3}\right)(P<0.001)$. 


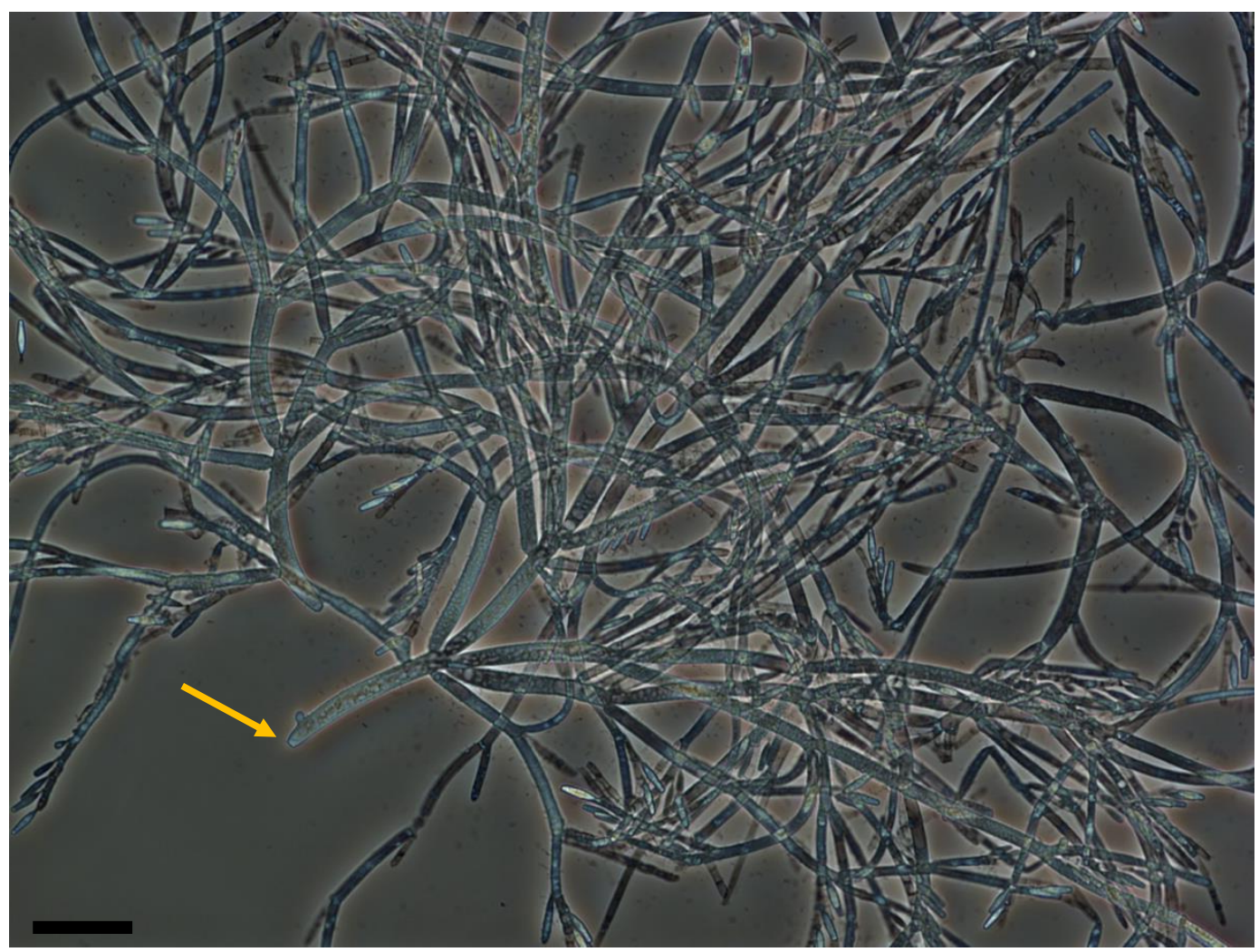

Plate 2.1:Zancudomyces culisetae: densely branched thallus, some with fertile tips, and with a simple holdfast (arrow) (ID-333MM1). Bar $=50 \mu \mathrm{m}$. 


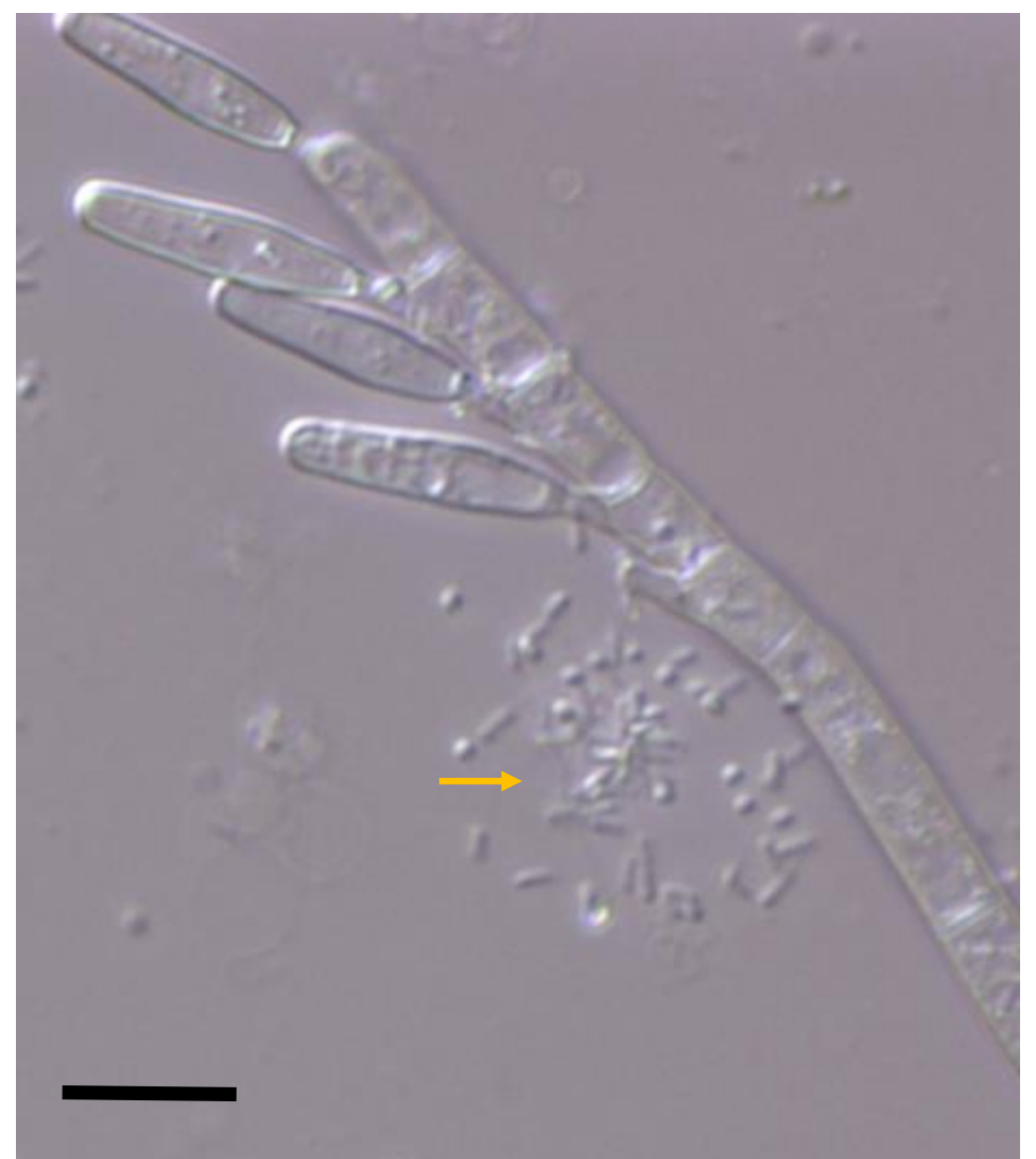

Plate 2.2: Zancudomyces culisetae, with four trichospores attached each to a generative cell at the fertile tip of the thallus. Arrow points to bacteria. (NIC*). Bar $=10 \mu \mathrm{m}$. ( ${ }^{*}$ Nomarski Interference Contrast) 


\section{REFERENCES}

Anderson JF and Main AJ. 2006. Importance of vertical and horizontal transmission of West Nile Virus by Culex pipiens in the Northeastern United States. 194(12):1800.

Becker N, Petric D, Zgomba M, Boase C, Madon MB, Dahl C, Kaiser A. 2003. Mosquitoes and their control. Kluwer Academic/Plenum Publishers, New York.

Bench ME and White MM. 2012. New species and first records of trichomycetes from immature aquatic insects in Idaho. Mycologia. 104(1):295-312.

Brothers DR. 2003. Mosquitoes of Idaho an introductory guide to understanding them, their importance, and the control process. PROBES (Project for Research on Biological and Environmental Subjects).

Carpenter SJ and LaCasse WJ. 1955. Mosquitoes of North America (North of Mexico). University of California Press. pp. 82-97.

Evans E. 1965. Survival of Fomes annosus in infected roots in soil. Nature. 207:318-319.

Ficetola GF, Miaud C, Pompanon F, Taberlet P. 2008. Species detection using environmental DNA from water samples. Biology Letters. 4:423-425.

Giller PS and Malmqvist B. 1998. The Biology of streams and rivers. Oxford University Press. p. 18.

Gillespie JM, Barr WF, Elliot ST. 1994. Taxonomy and biology of the immature stages of species of Thaumalea occurring in Idaho and California (Diptera: Thaumaleidae). MYIA. 5:153-193. 
Hong TD, Ellis RH, Moore D. 1997. Development of a model to predict the effect of temperature and moisture on fungal spore longevity. Annals of Botany. 79:121-128.

Horn BW. 1989. Requirement for potassium and pH shift in host-mediated sporangiospore extrusion from trichospores of Smittium culisetae and other Smittium species. Mycological Research. 93(3):303-313.

Hibbett DS, Binder M, Bischoff JF, Blackwell M, Cannon PF, Eriksson OE, Hundorf S, James T, Kirk PM, Lücking R, Thorsten Lumbsch H, Lutzoni F, Matheny PB, McLaughlin DJ, Powell MJ, Redhead S, Schoch CL, Spatafora JW, Stalpers JA, Vilgalys R, Aime MC, Aptroot A, Bauer R, Begerow D, Benny GL, Castlebury LA, Crous PW, Dai YC, Gams W, Geiser DM, Griffith GW, Gueidan C, Hawksworth DL, Hestmark G, Hosaka K, Humber RA, Hyde KD, Ironside JE, Köljalg U, Kurtz-man CP, Larsson KH, Lichtwardt RW, Longcore J, Miadlikowska J, Miller A, Moncalvo JM, Mozley-Stan dridge S, Oberwinkler F, Parmasto E, Reeb V, Rogers JD, Roux C, Ryvarden L, Sampaio JP, Schüßler A, Sugiyama J, Thorn RG, Tibell L, Untereiner WA, Walker C, Wang Z, Weir A, Weiss M, White MM, Winka K, Yao YJ, Zhang N. 2007. A higher level phylogenetic classification of the Fungi. Mycological Research 122:509547.

Kim S-K and Adler PH. 2007. Size variation of trichospores of Harpella melusinae in larval black flies. Mycologia. 99(3):351-355.

Kottek M, Grieser J, Beck C, Rudolf B, Rubel F. 2006. World Map of the Köppen-Geiger climate classification updated. Meteorologische Zeitschrift. 15(3):259-263.

Labeyrie ES, Molloy DP, Lichtwardt RW. 1996. An investigation of Harpellales (Trichomycetes) in New York state blackflies (Diptera: Simuliidae). Journal of Invertebrate Pathology 68:293-298. 
Lichtwardt RW, Williams MC, Ferrington Jr LC Jr, Hayford BL. 1997.

Harpellales: generic confusion due to precocious development. Mycologia. 89(1):109-113.

Lichtwardt RW, Cafaro MJ, White MM. 2004. The Trichomycetes: fungal associates of arthropods. [Internet]. Available from: http://www.nhm.ku.edu/ fungi/Monograph/Text/Mono.Htm

Lichtwardt RW. 1963. Unpublished data notebook, HAW 1-21. Housed in Merlin White's professional library. Department of Biological Sciences. Boise State University.

Lichtwardt RW. 1986. The Trichomycetes: fungal associates of arthropods. Springer-Verlag. pp. 3-332.

Lichtwardt RW. 2007. Lichtwardt's Keys to the Trichomycetes [Internet]. Available from: http://keys.lucidcentral.org/key-server/data/0b08020cOf0c-4908-8807-030c020a0002/media/Html/index.html

Mijung K, Robich RM, Rinehart JP, Denlinger DL. 2006. Upregulation of two actin genes and redistribution of actin during diapause and cold stress in the northern house mosquito, Culex pipiens. Journal of Insect Physiology. $52: 1226-1233$

Mackie G. 2004. Applied Aquatic Ecosystem Concepts. Kendall/Hunt Publishing Company. pp. 44-374.

Misra JK and Horn BW. 2001. Trichomycetes and Other Fungal Groups. Science Publishers, Inc. pp. 3-21.

Moss ST and Descals E. 1986. A previously undescribed stage in the life cycle of Harpellales (Trichomycetes). Mycologia. 78(2):213-222.

Moss ST and Taylor MR. 1994. An ovarian stage in species of the Harpellales (Trichomycetes). Abstract, V International Mycological Congress, Vancouver, p. 149. 
Moss ST. 1998. Harpellales (Trichomycetes); mycobionts of insecta. Botanical Journal of Scotland. 50(2):137-152.

NACSE. 2017. Northwest Alliance for Computational Science and Engineering. PRISM Climate Group, Oregon State University, http://prism.oregonstate.edu Map created <08/29/2017>.

Rizzo A and Pang K. 2005. A zygosporic stage of the Harpellales (Trichomycetes, Zygomycota) associated with simuliid egg masses. Mycological Research. 109(3):266-270.

Spatafora JW, Chang Y, Benny GL, Lazarus K, Smith ME, Berbee ML, Bonito G, Corradi N, Grigoriev I, Gryganskyi A, James TY, O’Donnell K, Roberson RW, Taylor TN, Uehling J, Vilgalys R, White MM, Stajich JE. 2016. A phylum-level phylogenetic classification of zygomycete fungi based on genome-scale data. Mycologia 108:5 1028-1046.

Spielman A, D’Antonio M. 2001. Mosquito: a natural history of our most persistent and deadly foe. New York: Hyperion. 110-115.

Statzner B, and Borchardt D. 1994. Longitudinal patterns and processes along streams: modelling ecological responses to physical gradients. Aquatic ecology. Proc. BES Symposium, Cork, 1992. 113-140.

Strongman DB, Wang J, Xu S. 2010. New trichomycetes from western China, Mycologia. 102:1, 174-184.

Tuzet O, Rioux JA, Manier JF. 1961. Rubetella culicis (Tuzet et Manier 1947), Trichomycète rameux parasite de l'ampoule rectale des larves de Culicides (morphologie et spécificité). Vie et Milieu. 12:167-187.

Valle LG, and Santamaria S. 2004. The genus Smittium (Trichomycetes, Harpellales) In the Iberian Peninsula. Mycologia. 96(3):682-701.

Vojvodic S and McCreadie JW. 2009. Morphological differences of symbiotic fungi Smittium culisetae (Harpellales: Legeriomycetaceae) in different Dipteran hosts. Mycological Research. 113:967-972. 
Wang Y, Tretter ED, Lichtwardt RW, White MM. 2013. Overview of 75 years of Smittium research, establishing a new genus for Smittium culisetae, and prospects for future revisions of the 'Smittium' clade. Mycologia. 105(1):90-111.

White MM, Siri A, Lichtwardt RW. 2006. Trichomycetes insect symbionts in Great Smoky Mountains National Park and vicinity. Mycologia. 98:2, 333-352.

Williams MC. 1983. Spore longevity of Smittium culisetae (Harpellales, Legeriomycetaceae). Mycologia. 75:171-174.

Wilson E. 2013. Evaluating the effects of fungicides and other pesticides on nontarget gut fungi and their aquatic insect hosts. MS Thesis, Department of Biological Sciences, Boise State University, Boise, ID, USA. Appendix C 
APPENDIX 
An extended table of survey results for Idaho 


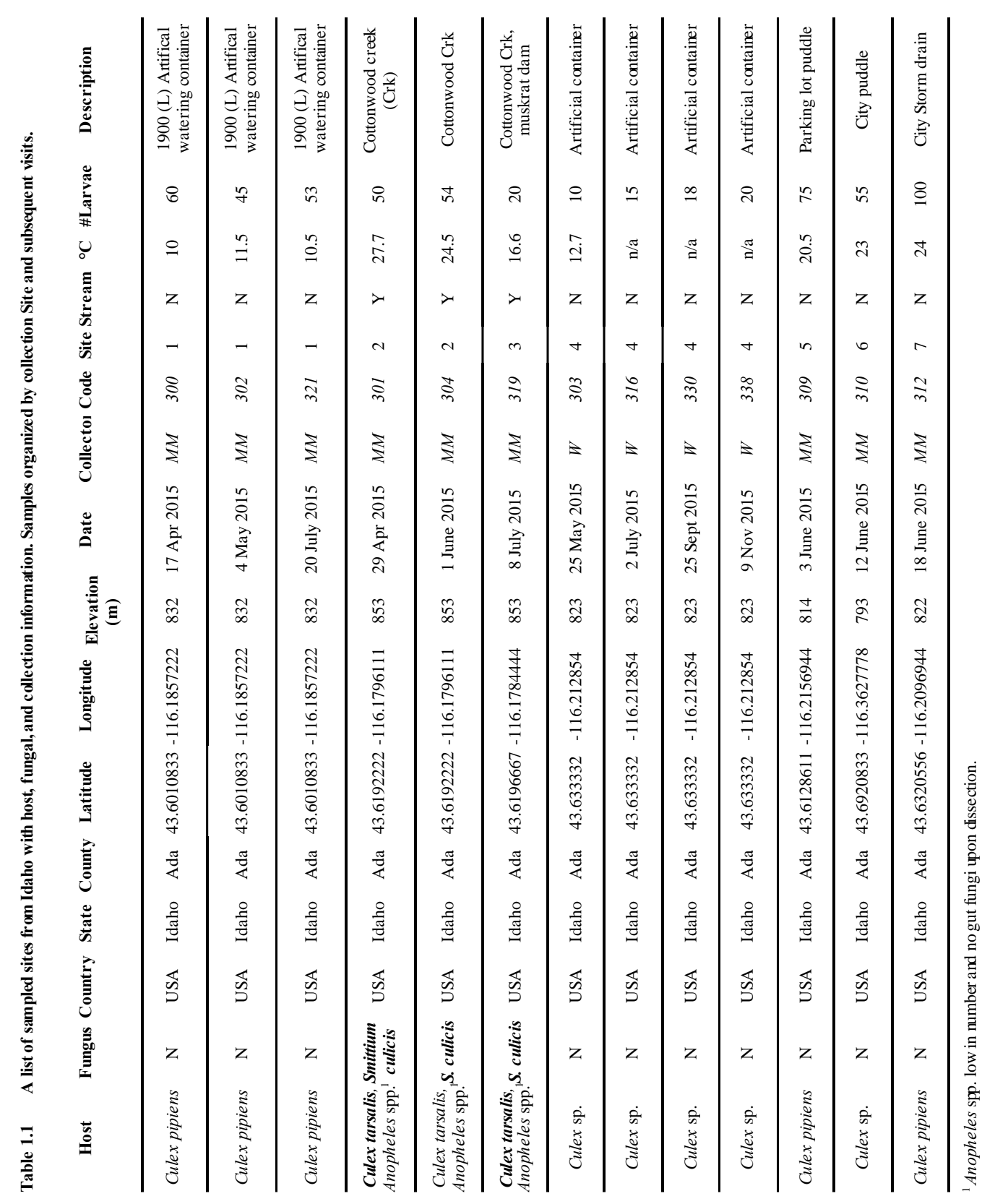




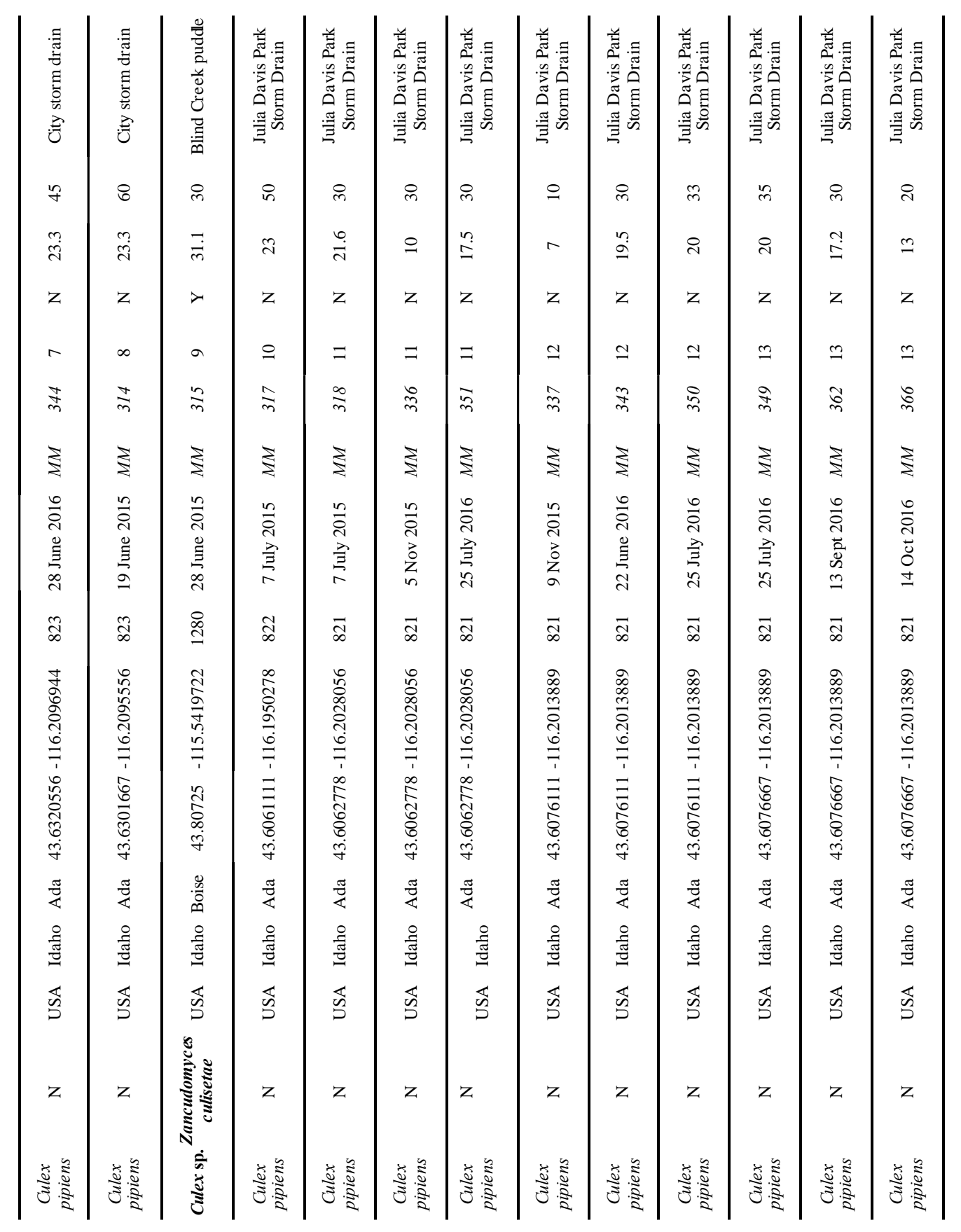




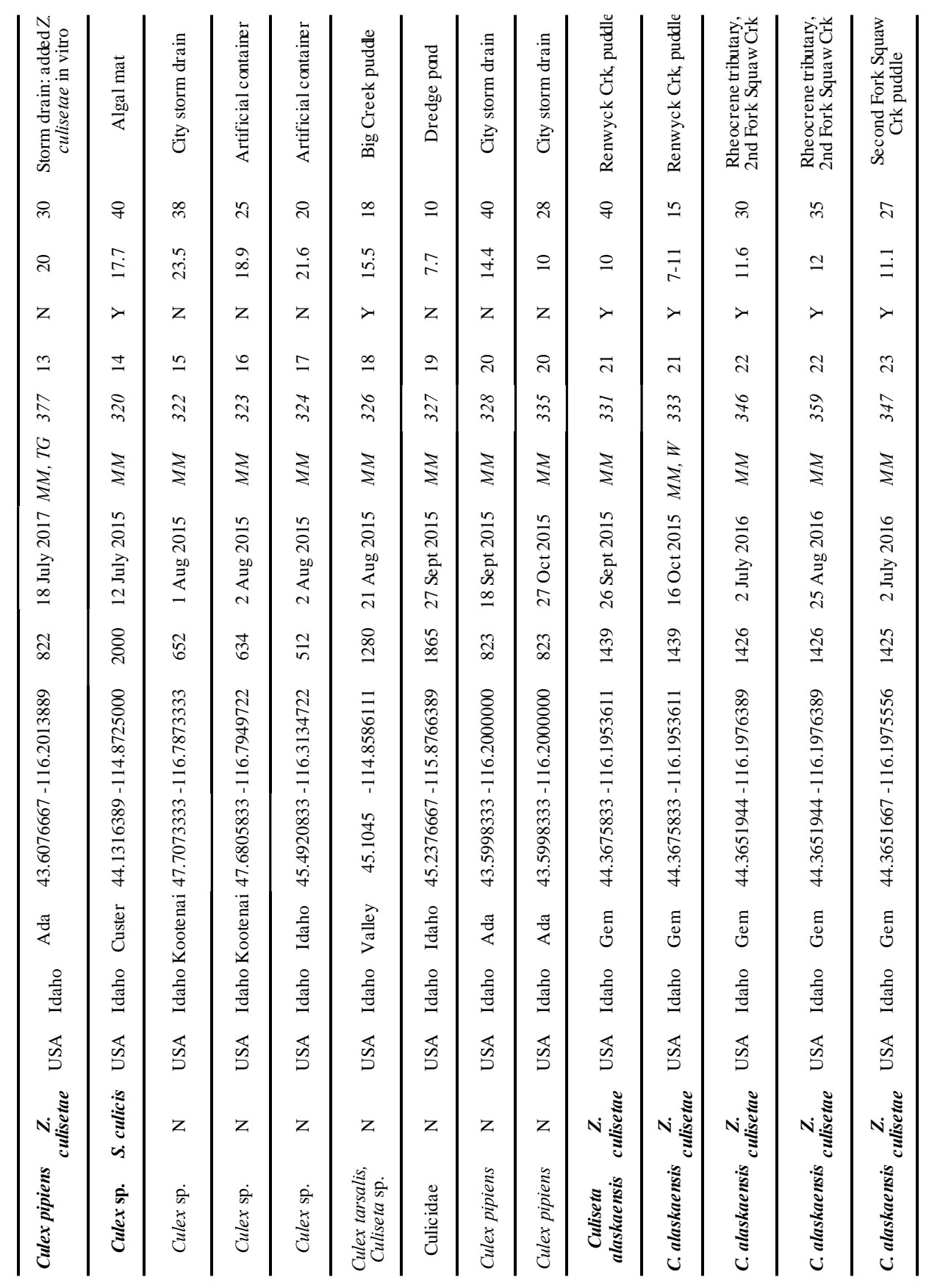




\begin{tabular}{|c|c|c|c|c|c|c|c|c|c|c|c|c|c|}
\hline 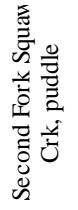 & 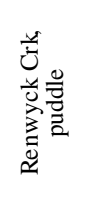 & 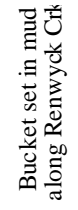 & 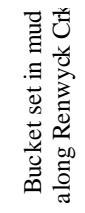 & 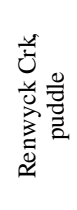 & 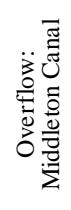 & 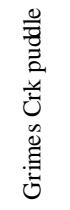 & 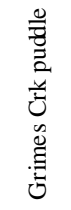 & 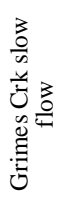 & 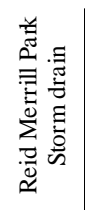 & 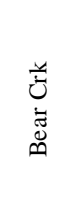 & 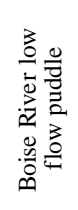 & 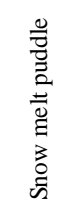 & 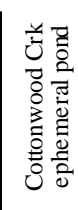 \\
\hline$\tilde{m}$ & $\ddot{\sim}$ & i & in & $\stackrel{\sim}{i}$ & p & ה & $\infty$ & 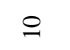 & $\cong$ & m & i & $\stackrel{\sim}{\sim}$ & $\overline{6}$ \\
\hline$\stackrel{\sim}{\stackrel{\sim}{m}}$ & 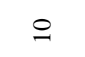 & 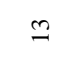 & 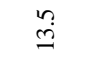 & ֵֶ) & 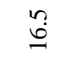 & $\stackrel{n}{I}$ & $\stackrel{\sim}{\underset{\sim}{\sim}}$ & त) & $\cong$ & $\tilde{I}$ & $\stackrel{+}{ \pm}$ & $\infty$ & 4 \\
\hline$\lambda$ & $\lambda$ & $\lambda$ & $\lambda$ & $\lambda$ & $\succ$ & $\lambda$ & $\lambda$ & $\lambda$ & z & $\lambda$ & $\lambda$ & $z$ & $z$ \\
\hline 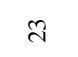 & $\stackrel{ \pm}{\sim}$ & n & ñ & $\stackrel{\text { N }}{ }$ & $\hat{\sim}$ & $\stackrel{\infty}{\sim}$ & ते & p & $\bar{m}$ & లె & $m$ & मे & $m$ \\
\hline$\stackrel{\infty}{m}$ & $\stackrel{\infty}{d}$ & હે & iे & $\stackrel{n}{n}$ & $\tilde{m}$ & ळे & $\stackrel{\circ}{\circ}$ & $\bar{b}$ & $\tilde{n}$ & $\stackrel{m}{n}$ & 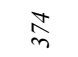 & i & $\hat{m}$ \\
\hline 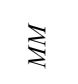 & $\xi$ & 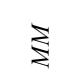 & 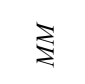 & $\Xi$ & $\begin{array}{l}\geqq \\
\Xi\end{array}$ & 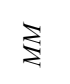 & 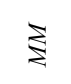 & $\stackrel{\Sigma}{\Sigma}$ & 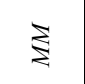 & 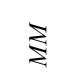 & 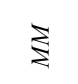 & 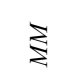 & \\
\hline 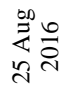 & 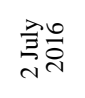 & 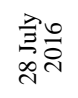 & 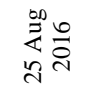 & $\begin{array}{l}\text { 空。 } \\
\text { 品 }\end{array}$ & $\begin{array}{l}\vec{s} n \\
\dot{0} \\
+\bar{c}\end{array}$ & 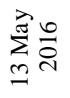 & 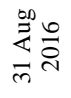 & 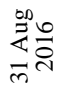 & $\begin{array}{l}\overrightarrow{\mathrm{s}} \\
\stackrel{0}{0}\end{array}$ & 言完 & 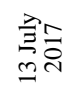 & 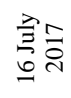 & 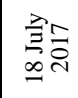 \\
\hline$\stackrel{\sim}{\text { I }}$ & $\begin{array}{l}\infty \\
\stackrel{\infty}{\exists}\end{array}$ & 怘 & 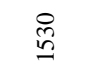 & 产 & 号 & $\stackrel{\infty}{\Xi}$ & $\stackrel{n}{\Xi}$ & $\stackrel{\circ}{\varrho}$ & $\stackrel{\infty}{\circ}$ & 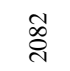 & $\stackrel{\infty}{\infty}$ & $\underset{\text { ते }}{+}$ & $\stackrel{\infty}{\infty}$ \\
\hline 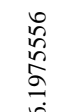 & $\begin{array}{l}\frac{J}{2} \\
\frac{\partial}{2} \\
\stackrel{2}{0}\end{array}$ & $\begin{array}{l}\stackrel{0}{ } \\
\infty \\
\infty \\
\infty \\
\infty\end{array}$ & 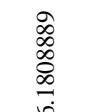 & 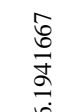 & 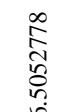 & 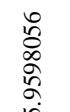 & 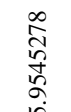 & 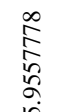 & 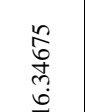 & $\begin{array}{l}\text { స̃ } \\
\text { ડे } \\
\text { ปे }\end{array}$ & 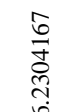 & $\begin{array}{l}\circ \\
\stackrel{े}{\sigma} \\
\stackrel{+}{\sigma} \\
\sigma\end{array}$ & 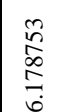 \\
\hline$\dddot{6}$ & $\stackrel{6}{7}$ & $\stackrel{0}{7}$ & $\dddot{6}$ & 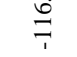 & $\stackrel{0}{7}$ & $\stackrel{n}{=}$ & $\stackrel{n}{\beth}$ & $\stackrel{n}{7}$ & $\overline{7}$ & $\stackrel{n}{7}$ & $\dddot{6}$ & $\frac{n}{7}$ & $=$ \\
\hline 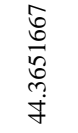 & 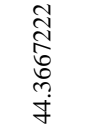 & \begin{tabular}{l} 
ป̃ \\
n. \\
- \\
\multirow{J}{*}{}
\end{tabular} & \begin{tabular}{l} 
त̃ \\
ñ \\
$\hat{n}$ \\
\multirow{7}{*}{}
\end{tabular} & 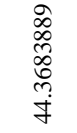 & 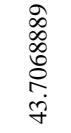 & 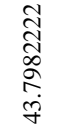 & 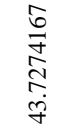 & 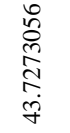 & 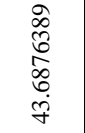 & 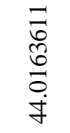 & 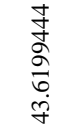 & 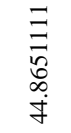 & 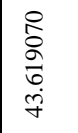 \\
\hline ฮั & छ̃ & ర్ & छี & हี & $\frac{\pi}{4}$ & 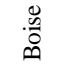 & 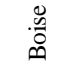 & $\begin{array}{l}.00 \\
0 \\
0\end{array}$ & $\frac{\pi}{<}$ & $\begin{array}{l}\mathscr{m} \\
.0 \\
.0\end{array}$ & $\frac{\pi}{4}$ & $\frac{\overrightarrow{0}}{\bar{\nu}}$ & $\frac{\pi}{4}$ \\
\hline 鴣 & 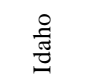 & 鴣 & 总 & 总 & 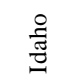 & $\begin{array}{l}\text { 䔍 } \\
\text { (n) }\end{array}$ & 胥 & 鴣 & 䔍 & 营 & $\begin{array}{l}\text { 丞 } \\
\end{array}$ & $\begin{array}{l}\text { 䔍 } \\
\text { (n) }\end{array}$ & 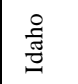 \\
\hline 芯 & 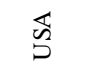 & 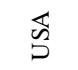 & 芯 & $\overleftrightarrow{s}$ & 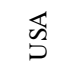 & 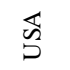 & 崩 & 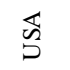 & 芯 & 盛 & 芯 & 尚 & 品 \\
\hline 气ัँ & 气ू 气 & $z$ & z & 䒕 & z & 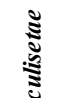 & 气ั้ & $\mathrm{z}$ & $z$ & 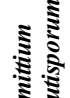 & z & $\mathrm{z}$ & $z$ \\
\hline 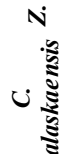 & 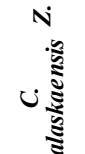 & ن & ن & ن & 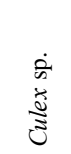 & 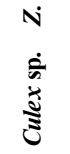 & 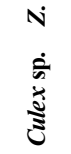 & نू & 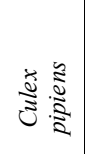 & $\frac{5}{5}$ & 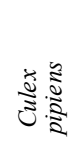 & को & 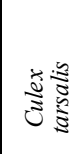 \\
\hline
\end{tabular}


An extended table of Renwyck Creek collections and further observations 


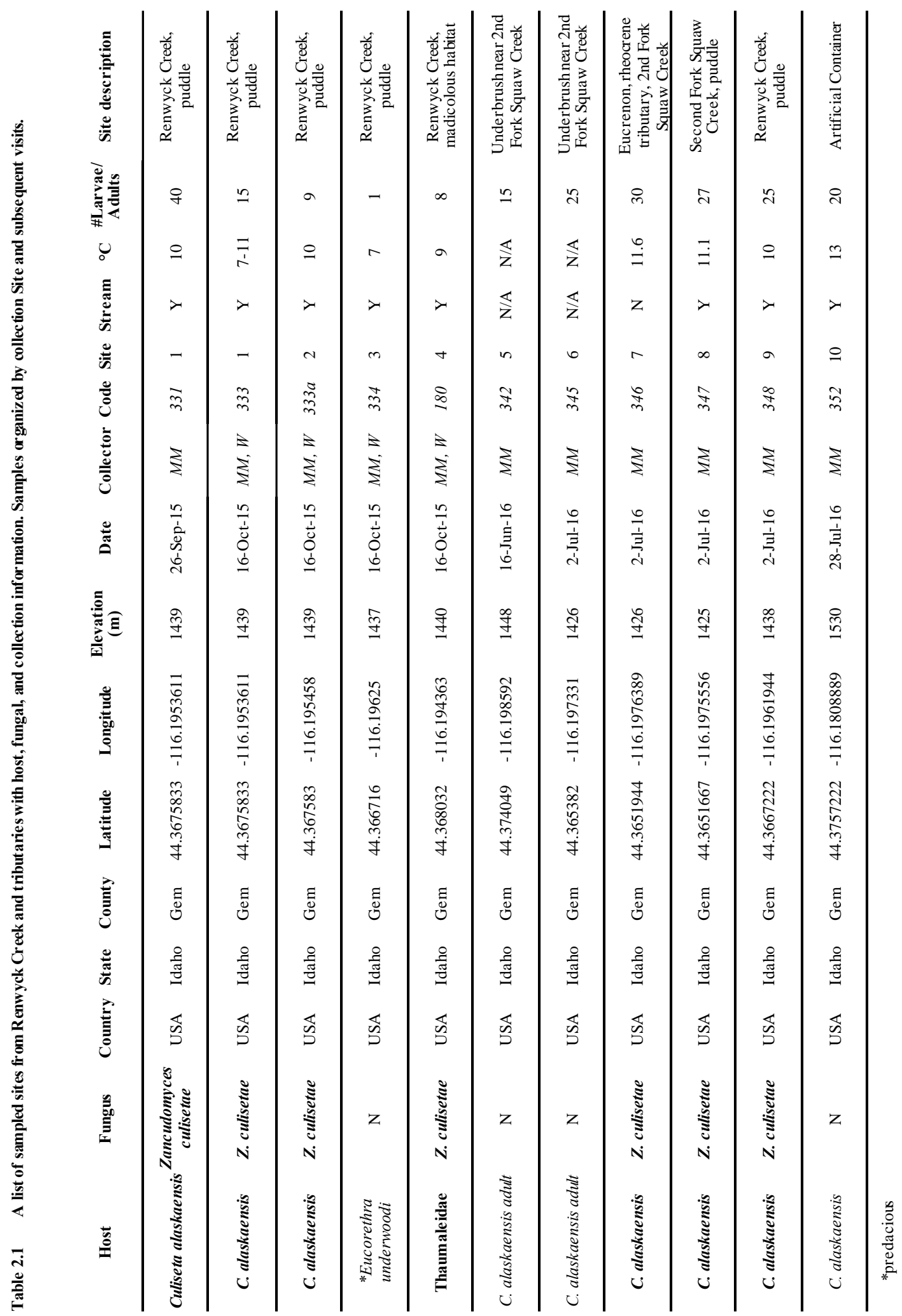




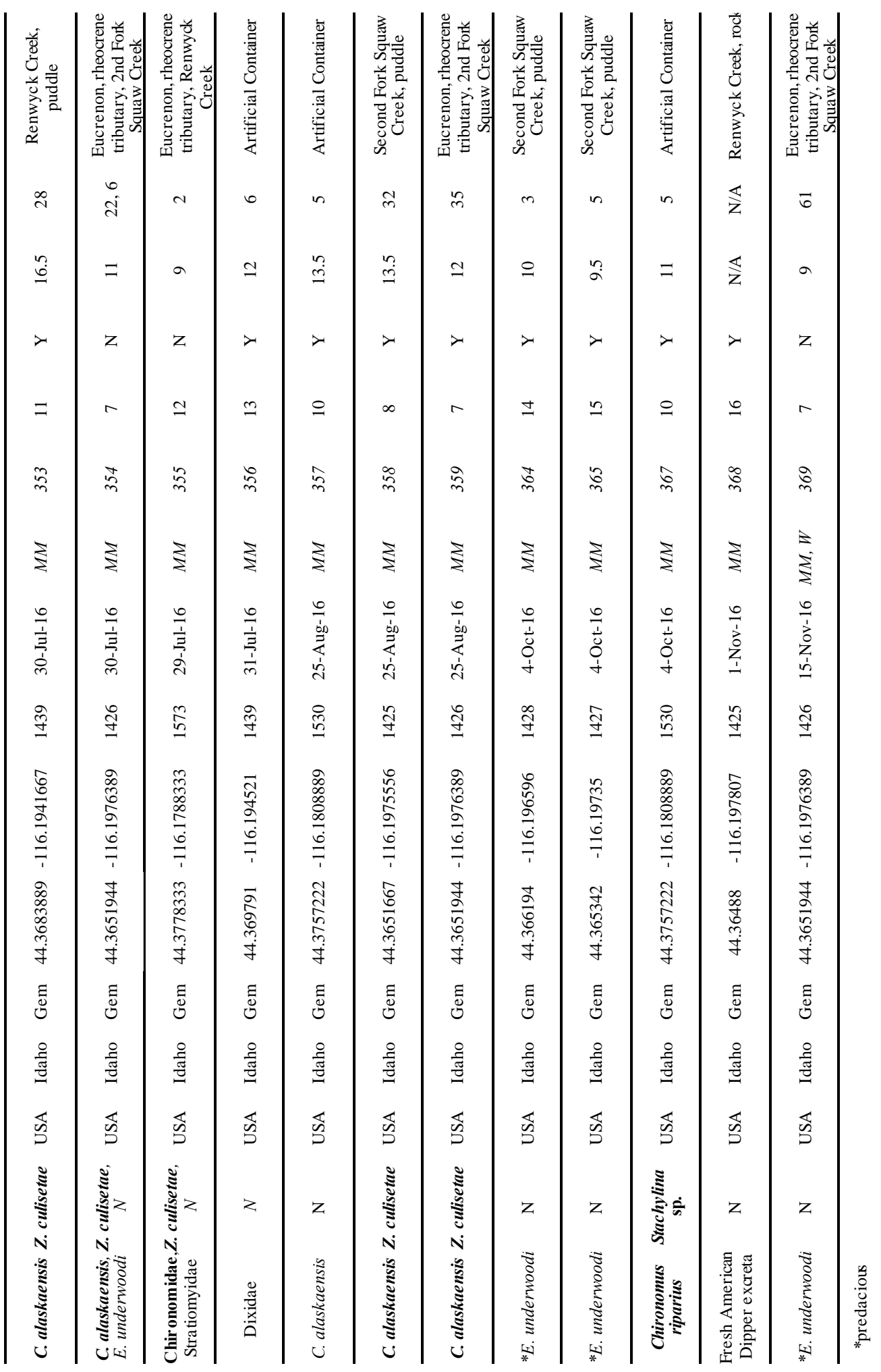




\section{$\underline{\text { Addendum }}$}

During my study, American Dipper (Aves: Cinclus mexicanus) excreta was also explored as a means for transporting spores along the creek gradient. Although spores were not found via light microscopy from fresh excreta (unpublished data), it remains possible that these birds, which bathe and eat regularly from Renwyck Creek, are a means for transportation of spores on their feet, feathers, or in their excreta as they travel regularly up the creek. Given the difficulties associated with working on natural avian systems to address a question of this nature, perhaps the first step would be to study its potential as an explanation using captive birds in a laboratory setting. Such an experiment would involve feeding live cultures of gut fungi to the captive birds, examining their excreta for viable fungal spores, and testing the ability of these birds to transfer fungi from one site to another. Bird motility also brings to attention the possibility of other aquatic animals as possible mechanisms for spore transfer between sites. Fish and or frogs may give a great advantage to gut fungi dispersal, having consumed host arthropods and then transporting gut fungi up and down the stream system releasing spores via their excreta.

In short, there may be multiple methods of spore shuttling including through the water column, hitchhiking on or in an animal, and lastly vertical transmission through a mosquito or other dipteran host. Exploring further each of these possible transfer mechanisms would help to unravel Harpellales spore dispersal and life cycle patterns. The presence of Stachylina sp. gut fungi in Chironomus riparius collected from artificial containers suggests that midges may be a good source for advancing knowledge on vertical transmission. 
Lastly, collection ID-301 (site 2; Table 1.1), provided insight into spore longevity and should be further studied with various temperature and moisture treatments similar to Moss (1983). The ID-301 collection bag, with its water and detritus, was stored for 1 year at $4^{\circ} \mathrm{C}$ (April 2015-April 2016). Sterile southern house mosquito larvae, Culex quinquefasciatus, obtained from Benzon Research were added to the collection bag of ID-301 and became readily infested with Smittium culicis. Control C. quinquefasciatus did not become infested with any gut fungi. This demonstrates that under the right conditions, trichospores and or zygospores can survive outside the host for long periods of time. 